\title{
CALCULATION OF RADIATION FIELDS IN THE ATMOSPHERE AND COMPARISON TO EXPERIMENTAL DATA
}

\author{
S. Roesler, W. Heinrich \\ Universität Siegen, Fachbereich Physik, D-57068 Siegen, Germany \\ and \\ H. Schraube \\ GSF-Forschungszentrum für Umwelt und Gesundheit, Institut für Strahlenschutz, \\ D-85758 Neuherberg, Germany
}

\begin{abstract}
In this paper spectra of secondary hadrons are determined in the atmosphere at various $5 w / 448$ depths down to sea level. The calculations are performed by means of the Monte Carlo code FLUKA together with cosmic ray environmental models which describe the spectra of primary cosmic ray particles. We discuss details of the calculations and the dependences of the results on the cosmic ray primary spectrum, the atmospheric depth, and the geographical location. Special emphasis is put on comparisons of calculated and measured hadron spectra including an experiment which was recently performed at the Zugspitze. It is shown that FLUKA may serve as important tool for the estimation of the radiation environment in the atmosphere.
\end{abstract}

Submitted for publication in

Radiation Research 


\section{Introduction}

Cosmic rays with energies which are sufficiently high to penetrate the geomagnetic shielding of the earth initiate particle showers in the atmosphere and shielding materials of aircrafts. Detailed studies of the cascade processes (see [1] and references therein) are necessary for the determination of risk relevant quantities $[2,3]$ for air crews and other frequently flying individuals. An essential contribution to the radiation risk is caused by secondary neutrons. Therefore, the present discussions of atmospheric hadron spectra are mainly focused on neutrons.

It was pointed out in Refs. $[2,3]$ that measurements of neutron-spectra at different altitudes in the atmosphere suffer from technical difficulties, like non-stationary experimental positions for measurements in aircrafts, insufficient knowledge of the instrumental responses or from poor statistics. At present, experimental investigations are still far from giving a detailed description of hadron spectra in the atmosphere. Studies have therefore in addition to rely on theoretical models describing interaction processes and transport mechanisms of particles in matter. The predictions of those models are carefully tested against measurements at high energy accelerators and of cosmic ray air showers, like deep underground muons or Extensive Air Showers $[4,5,6,7,8,9,10,11,12,13,14]$. This fact justifies their application to aspects of particle production in the atmosphere and shielding materials where experimental information is still sparse, like it is the case for neutron spectra. However, it has to be noted that even though the interactions of cosmic ray particles with nuclei of the atmosphere or of the shielding materials together with the transport and the subsequent collisions of the secondary particles are well understood, considerable uncertainties remain. They arise from insufficient knowledge of the "input" needed for the calculation as it is, for instance, the primary cosmic ray spectrum [15].

The present paper describes an attempt to extend the studies of cosmic ray cascades to hadron, especially neutron, spectra in the atmosphere by applying the Monte Carlo (MC) model FLUKA $[16,17,18]$. Beside other MC-particle transport codes like LAHET [19] or HETC [20], FLUKA is widely used in radiation shielding calculations. It proved to be very reliable in predicting stray radiation fields around high energy accelerators $[4,21]$ which are comparable to radiation fields in the atmosphere, and therefore it seems to us most suitable for our present investigation. The electromagnetic component of air showers will be subject to a forthcoming study. In Sect.2 we give a short account on FLUKA and the models it is based on. Furthermore, we discuss the geometrical treatment of the atmosphere and the spectra of primary cosmic ray particles entering the calculations. In Sect.3 we present calculated hadron spectra at different altitudes and for different geographical positions and times. In Sect. 4 the predictions of the model are compared to measurements including results of an experiment recently performed at the Zugspitze $[2,3]$. Finally, in Sect.5 we summarize our results. 


\section{Cosmic ray cascade calculations}

\subsection{The Monte Carlo model FLUKA}

FLUKA simulates the whole high energy hadronic and electromagnetic cascade from $\mathrm{TeV}$ to thermal energies. Applications range from detector design to low energy neutron and photon background estimation in underground neutrino detectors and to basic in-phantom dosimetry.

Hadronic interactions in FLUKA are simulated based on different models depending on the energies of the primary particles. The Dual Parton Model [22] is applied at energies above $5 \mathrm{GeV}$ while a model based on resonance production and decay is used at lower energies [23]. A pre-equilibrium-cascade model describes inelastic hadronic collisions in the intermediate energy range [24] and nuclear evaporation and $\gamma$-deexcitation models [25, 26] account for the emission of neutrons, protons, light nuclei, and gamma rays from the excited nuclei. Leptons may interact in photonuclear scattering processes. All these models were itself compared to results of measurements and describe successfully main aspects of high and low energy particle production (see [27] and references therein). Charged particles are transported applying an improved multiple scattering algorithm based on Moliére's theory of Coulomb scattering [28]. They suffer energy loss according to the Bethe-Bloch theory and due to bremsstrahlung and pair production. Neutrons below $20 \mathrm{MeV}$ are transported using a neutron cross section data library which was developed for FLUKA [29]. A Combinatorial Geometry package allows the definition of the geometry and the assignment of different materials to the regions of the problem to be investigated.

The calculations discussed in this paper were performed with the FLUKA-95 version of the code [30].

\subsection{A model for the atmosphere}

The atmosphere is defined by a stack of slabs with thicknesses which are increasing with the height above sea level. The thickness of any slab is chosen to correspond to a depth of $50 \mathrm{~g} / \mathrm{cm}^{2}$ making use of a fit to the vertical profile of the "standard atmosphere" by M. Shibata [31]

$$
h_{v}(\mathrm{~km})=\left\{\begin{aligned}
47.05-6.9 \ln X_{v}+0.299 \ln ^{2}\left(0.1 X_{v}\right) & : X_{v}<25 \mathrm{~g} / \mathrm{cm}^{2} \\
45.5-6.34 \ln X_{v} & : 25<X_{v}<230 \mathrm{~g} / \mathrm{cm}^{2} \\
44.34-11.861 X_{v}^{0.19} & : X_{v}>230 \mathrm{~g} / \mathrm{cm}^{2}
\end{aligned}\right.
$$

$X_{v}$ and $h_{v}$ are the vertical depth in the atmosphere (in $\mathrm{g} / \mathrm{cm}^{2}$ ) and height above sea level (in $\mathrm{km}$ ) with the top of the atmosphere limited at $2 \mathrm{~g} / \mathrm{cm}^{2}(\simeq 43.0 \mathrm{~km})$ and the bottom at $1000 \mathrm{~g} / \mathrm{cm}^{2}$ (corresponding approximately to the sea level). The profile of the atmosphere is shown in Fig.1. The dots represent the slab approximation. In each slab we assume a uniform density of air ${ }^{1}$ which we obtain from the thickness and the corresponding depth of the slab. The air is defined as a composition of nitrogen and oxygen of mass fractions of 0.76 and 0.24 ,

\footnotetext{
${ }^{1}$ We are aware that this assumption, especially for the uppermost slab, might be too simplified for other applications (for instance those sensitive to particle decays). However, it is sufficient within the scope of the present investigation
} 
respectively. Even though argon has a significant neutron capture cross section it has been omitted since due to its small mass fraction in air $(\approx 1.3 \%)$ the influence on the results is negligible.

The uncertainties within the results arising from the geometrical treatment are estimated to be minor as compared to the uncertainties within the primary cosmic ray spectra (see next Chapter).

\subsection{The spectra of primary cosmic ray particles}

A compilation of measured energy spectra below $50 \mathrm{GeV} /$ nucleon of cosmic ray particles in the absence of the geomagnetic field, is given together with fits to these data by Adams, Silberberg, and Tsao in [32]. We have adopted these fits in our procedure of sampling primary cosmic ray particles. Here, we only want to underline a few points which seem to be essential to us for the estimation of the uncertainties of our calculations. For further details we refer to $[32]$.

Cosmic rays mainly consist of hydrogen and helium nuclei (about $85 \%$ and $12.5 \%$, resp.). We take spectra of all nuclei with charges up to $Z=28$ (nickel) into consideration. Since in FLUKA nucleus-nucleus interactions and the transport of nuclei and fragments with $A>1$ are not treated consistently we have to employ the so-called "superposition model" in which it is assumed that a shower produced by a nucleus of mass $A$ is equivalent to the superposition of $A$ nucleon showers. In our case it means that the spectra of primary nuclei are rescaled according to charge and mass number and primary protons and neutrons are sampled correspondingly. Each shower starts at a depth calculated according to the proton-air cross section. With respect to the low relative abundances of nuclei with $A>1$ this might be sufficient for our present studies. However, as it is shown in [33] we might obtain cosmic ray showers with less fluctuations than measured. See also Refs. $[5,34]$ for a discussion on this subject.

As it is shown in Fig.2a the differential energy spectra below about $5 \mathrm{GeV} /$ nucleon are spread between two extremes which are determined by solar modulation depending on the level of solar activity. This modulation is characterized by a 11-years-cycle and can be roughly approximated by a sinusoidal fit [32]. In Fig.2b we show as an example the normalized monthly average neutron rate measured by the neutron monitor at Deep River (Ontario, Canada) during the past 40 years together with a sinusoidal fit. As it can be clearly seen, the fit may differ from the measured counting rate by up to $10 \%$ of the normalized rate, i.e. by about $60 \%$ of the total modulation effect. It follows, that when using the sinusoidal approximation of the 11-years-solar cycle an uncertainty of that order is entering calculations for geographical positions in the atmosphere with relatively weak geomagnetic field (such as positions close to the magnetic poles), i.e. where the rigidity cut is low. Therefore, in this work we use the primary spectrum corresponding to the actual Deep River neutron monitor counting rate for comparisons to experimental data, but have to rely on the sinusoidal fit for predictions of spectra at future times.

The geomagnetic shielding against low-energy cosmic ray particles is taken into account by a energy-cutoff which can be estimated using the dipole approximation of the magnetic field [35]. The rigidity threshold $R_{c}$ for a particle at a position $(r, \lambda)$ (in spherical geomagnetic coordinates, $\lambda$ being the zenith angle) moving into the direction $(\theta, \phi)$ (in spherical coordinates 
of a frame with its origin at the position of the particle and $\theta$ measured from the radial direction in the geomagnetic frame) is given by

$$
R_{c}(r, \lambda, \theta, \phi)=4 R_{c}^{V}(r, \lambda) \frac{1}{\left(1+\sqrt{1-\sin \theta \cos \phi \cos ^{3} \lambda}\right)^{2}}
$$

The rigidity thresholds for vertically incident particles $R_{c}^{V}(r, \lambda)=R_{c}(r, \lambda, \theta=0)$ were calculated by Shea et al. [36]. The effect of the geomagnetic shielding on the differential energy spectrum is shown in Fig.2a for the geographical position of the Zugspitze $\left(47.4^{\circ} \mathrm{N}, 11.0^{\circ} \mathrm{E}\right)$.

As it was noted in [15] measurements of primary proton and helium spectra performed with different instruments may differ by almost up to a factor of two. This fact leads to further uncertainties within our calculations.

For primary energies above $50 \mathrm{GeV} /$ nucleon we apply a power-law spectrum [37]

$$
\frac{d \Phi_{\text {prim }}(E)}{d E} \sim E^{-\gamma}, \quad \gamma=2.7
$$

which is normalized in order to obtain a smooth transition to the fits of Adams et al..

\subsection{Details of the calculations}

The FLUKA calculations are done on an event-by-event basis with one event defined as the complete cascade initiated by one primary cosmic ray particle in the atmosphere or in a shielding material. The energy-spectrum of a hadron of a given type obtained at a certain depth is therefore a superposition of energy-spectra of all the calculated events.

The technical complexity of sampling a primary cosmic ray particle arises from the following facts. In general one would have to sample the impact position of a primary particle on top of the atmosphere uniformly distributed over an area. The size of this area had to be sufficiently large in order to cover all secondary particle positions which the detector at a certain depth is sensitive to. However, due to the slab geometry this can be simplified by sampling primaries on top of the atmosphere at one and the same position and to "detect" the secondaries at the considered depth regardless of their positions. Furthermore, the energies of primary particles contributing to the secondary flux at the detector depth extend over several decades. Together with the power-law falling primary energy spectrum and a certain (limited) number of events this fact may induce large fluctuations within the results of the calculations if one samples the energy linearly from the whole range. The fluctuations can be reduced by subdividing the energy interval into "bands" [7] and summing up the partial results. To be definite, we subdivide the energy range of the primary spectrum between the lower cut which arises from the geomagnetic shielding and an upper cut, for most of the calculations set at $1300 \mathrm{GeV}$, into three bands. The limits between them are $20 \mathrm{GeV}$ and $100 \mathrm{GeV}$. In addition, this strategy has the advantage that for calculations at different input conditions (i.e. different solar modulation and geographical positions) only the calculation for the lowest band $\left(E_{\text {prim }}<20 \mathrm{GeV}\right)$ has to be repeated, since it is the only band sensitive to these input effects. Finally, the direction of incidence of the primary particle is isotropically distributed in solid angle. 
The upper energy limit deserves a further comment. In general, when comparing to measurements the upper energy cut depends on the energy range and on the time interval under analysis. The realization of the Dual Parton Model used in FLUKA gives a reasonable description of hadron-nucleus interactions up to hadron energies of about $20 \mathrm{TeV}$. At higher energies observables like particle multiplicities or transverse momenta at high $p_{\perp}$ might be underestimated. However, the higher the energy of the primary is the smaller is its weight which eventually enters the calculation, i.e. the smaller is its contribution to the hadron flux at energies of at least two orders of magnitude below the energy of the primary. In addition, the CPU-time needed to compute the cascade down to sea level and to thermal neutron energies is exponentially increasing with the primary energy. For predictions of hadron fluxes, especially at high depths, it is therefore necessary to estimate the contribution of high energy primaries to the observables under investigation carefully, in order to choose an upper energy cut which keeps the CPU-time within reasonable limits but still allows reliable predictions.

For each event we sample

- the energy of the primary particle uniformly distributed within the considered energy band,

- the identity of the primary (proton or neutron) according to their spectra replacing nuclei of mass $A$ by $A$ independently interacting nucleons, and

- a zenith angle from an isotropic angular distribution.

The weight of an event is obtained from (i) a weight factor proportional to the primary flux at the sampled energy, (ii) the width of the energy interval the primary is sampled from, (iii) the cosine of the zenith angle (measured from the vertical), and (iv) the inverse of the number of events sampled in total.

The interactions of charged secondary hadrons and their transport are simulated down to a lower energy limit of $1 \mathrm{keV}$ whereas those of neutrons are treated down to $0.41 \mathrm{eV}$. Leptons and neutrinos are not transported because only hadronic interactions are of interest in the present study.

The energy spectra of secondary hadrons are recorded at the boundaries between subsequent slabs on a logarithmic basis in energy and, for neutrons below $20 \mathrm{MeV}$, according to the multi-group structure of the neutron cross section data library [29].

\section{$3 \quad$ Hadron spectra in the atmosphere}

\subsection{The dependence on the atmospheric depth}

In Fig. 3 we present secondary neutron (a) and charged hadron (predominantly protons and charged pions) energy spectra (b) calculated with FLUKA for depths of $200 \mathrm{~g} / \mathrm{cm}^{2}, 500 \mathrm{~g} / \mathrm{cm}^{2}$, and $900 \mathrm{~g} / \mathrm{cm}^{2}$ of air corresponding to heights above sea level of $11900 \mathrm{~m}, 5700 \mathrm{~m}$, and $1150 \mathrm{~m}$, respectively. The input spectrum of primary particles entering the calculations corresponds to the average solar activity in May 1995 and the geographical position of the Zugspitze (see Fig.2a). 
The shape of the neutron spectra shown in Fig.3a is characterized by a $E^{-\gamma}$-dependence below $50 \mathrm{keV}$ and, due to the lethargy-representation of the spectra, two "peaks" at energies of about $1 \mathrm{MeV}$ and $100 \mathrm{MeV}$. The $E^{-\gamma}$-dependence with $\gamma$ being about 0.92 (indicated in Fig.3a by a dotted line fitted to the low energy part of the spectrum at $200 \mathrm{~g} / \mathrm{cm}^{2}$ ) is caused by neutron slowing-down processes ${ }^{2}$, neutron absorption and is modified by the energy dependence of the neutron scattering cross sections. At higher energies, "neutron sources" dominate the spectral shape. The enhanced spectral neutron fluence rate around $1 \mathrm{MeV}$ arises from the evaporation of neutrons from excited residual nuclei. The spectral substructure clearly reflects the energy-dependence of the experimental low energy neutron cross sections which enter the FLUKA calculations [29]. In contrast, at energies of about $100-300 \mathrm{MeV}$ the neutron-air reaction cross sections are characterized by a broad minimum with a steep rise towards lower energies. Since the neutron spectrum is decreasing with increasing energy the enhanced spectral neutron fluence rate appears at slightly smaller energies of around 80 to $100 \mathrm{MeV}$. We note, that the shape of the neutron spectra below $1 \mathrm{GeV}$ does not depend on the depth after the cascade in the atmosphere has reached its maximum, i.e.

$$
\frac{d \Phi_{\mathrm{n}}\left(E, X_{v}\right)}{d E}=f\left(X_{v}\right) \frac{d \Phi_{\mathrm{n}}(E)}{d E}, \quad X_{v}>100 \mathrm{~g} / \mathrm{cm}^{2}
$$

The dependence of the fluence rate on the atmospheric depth is shown in Fig.4. Again, we give neutron and charged hadron fluence rates separately. The neutron fluence rate shows a maximum at about $100 \mathrm{~g} / \mathrm{cm}^{2}$ followed by an exponential decrease towards larger depths. The slope is slightly increasing depending on the lower limit in depth above which the results are fitted by the exponential. From a fit $\Phi_{n}\left(X_{v}\right)=\Phi_{n 0} \exp \left(-\beta X_{v}\right)$ to the fluence rates we obtain $\beta=0.0057 \pm 0.0004$ and $\beta=0.0064 \pm 0.0004$ for $X_{v}>400 \mathrm{~g} / \mathrm{cm}^{2}$ and $X_{v}>$ $600 \mathrm{~g} / \mathrm{cm}^{2}$, respectively. The latter is close to the value of $\beta=0.00721 \pm 0.00001$ deduced from measurements of the neutron monitor in Kiel, Germany for the same time (May 1995) [2].

\subsection{Influence of solar activity and geomagnetic shielding}

In order to demonstrate the influence of the level of solar activity and of the geomagnetic cutoff to the results we calculate hadron fluence rates for three extreme conditions: (i) geomagnetic pole and solar minimum (no geomagnetic shielding, maximum primary flux), (ii) geomagnetic pole and solar maximum, and (iii) geomagnetic equator (maximum shielding) ${ }^{3}$. The corresponding neutron fluence rates at different depths in the atmosphere are shown in Fig.5. By comparing the three cases we conclude that the neutron fluence rate at a certain depth may vary within a factor of two due to solar modulation but may increase by up to a factor ten when moving from the geomagnetic equator towards the poles. The maximum variation decreases with increasing depth, which means, for instance for the geomagnetic shielding effect, it decreases from a factor of ten at airplane altitudes $\left(\approx 200 \mathrm{~g} / \mathrm{cm}^{2}\right)$ to a factor of four at sea level. As it is indicated in Fig.5 by straight lines it follows from the fact, that the neutron

\footnotetext{
${ }^{2}$ In an infinite nonabsorbing medium with neutron scattering cross sections being independent of energy the spectrum would exactly go as $1 / E$

${ }^{3}$ Due to the relatively high lower cutoff in energy of the primary particles the solar modulation has no influence on the primary spectrum in this case
} 
fluence rate decreases faster for lower geomagnetic cutoffs. The reason is obvious: Only the low-energy part of the primary spectrum is affected by the geomagnetic shielding. It enters the calculation with a high flux increasing the overall hadronic activity, but is absorbed at lower depths than the primaries with higher energies. In Fig.6 we show the depth dependence of the charged hadron fluence rate obtained with the same three extreme primary spectra. In difference to the neutron fluence rates, here, the effects of solar activity and geomagnetic shielding are less pronounced; they even vanish at sea level. Charged particles suffer ionization energy losses and, in case of pions, they may decay, leading to the observed differences. Again, we note that an exact treatment of pion decay processes would require more slabs at low depths.

\section{Comparison of calculations to experimental data}

A collection of cosmic ray related measurements and calculations performed until 1984 can be found in [38]. In this Section we present comparisons of FLUKA-calculations to results of some of these measurements and to results of recent measurements performed on top of the Zugspitze, Germany and from the KASKADE-experiment in Karlsruhe, Germany.

\subsection{Differential energy spectra of neutrons}

In order to test spectrometry systems for air borne experiments and to collect more experimental information on neutron spectra in the atmosphere a measurement has been performed on top of the Zugspitze (2963m) in May 1995 [2]. Employing a Bonner Sphere Spectrometer and two different algorithms for unfolding the neutron spectrum from the count rates, the spectral neutron fluence rate from thermal energies up to $1 \mathrm{GeV}$ was obtained for a reference depth of $700 \mathrm{~g} / \mathrm{cm}^{2}$ [3]. In Fig.7a we compare these data to results obtained with FLUKA. The primary cosmic ray flux entering the calculations was chosen to correspond to the conditions during the measurements. At the geographical location of the Zugspitze, $47^{\circ} \mathrm{N} 11^{\circ} \mathrm{E}$, the atmosphere is shielded against primaries with energies below about $3.5 \mathrm{GeV}$ (see Fig.2a), the influence of the solar modulation being relatively unimportant. As mentioned in Sect.3.1 the shape of the spectrum below $1 \mathrm{GeV}$ does not change with depth. Since we are interested in a detailed comparison of the shape of the calculated and measured spectra, statistical uncertainties should be kept as small as possible. Therefore, in Fig.7a we show the spectrum calculated for a depth of $200 \mathrm{~g} / \mathrm{cm}^{2}$ normalized to the fluence rate at $700 \mathrm{~g} / \mathrm{cm}^{2}$ (see Fig.4) and integrated over the whole solid angle of $4 \pi$. The errorbars represent the statistical uncertainties. We underline again, that this procedure is only possible for energies below $1 \mathrm{GeV}$. If we were interested in comparing the high energy tail of the spectrum, the spectral fluence rate would be overestimated by rescaling smaller up to larger depths. For energies above $1 \mathrm{GeV}$ the calculated spectral fluence rates at $700 \mathrm{~g} / \mathrm{cm}^{2}$ are given.

Comparing the measured spectrum with the FLUKA results we note the following differences. Below $10 \mathrm{keV}$ the unfolded spectrum is lower than the calculated one by a factor of about two to three, the slope being steeper in the unfolded spectrum. The latter may arise from the fact that a $1 / E$-dependence was assumed within the guess-spectrum for the 
unfolding-procedure. As discussed in Sect.3.1 this might be a somewhat simplifying assumption, since absorption and energy-dependent cross sections lead to a lower slope [39, 40]. Above $10 \mathrm{keV}$ both spectra are characterized by two clearly pronounced neutron enhancements, as they were predicted in earlier cosmic ray cascade calculations by Armstrong et al. [11] and Merker [12] and also observed in stray radiation fields around high energy accelerators [4]. From the calculations the "peak" at $1 \mathrm{MeV}$ is again predicted to be higher with a more steady and smooth transition to the $E^{-\gamma}$-behaviour at lower energies than measured. The latter might be again due to the specific shape of the guess-spectrum which went into the unfolding procedure and is not necessarily a physical behaviour. As mentioned earlier, substructures inherent to the calculated spectrum between $100 \mathrm{keV}$ and $20 \mathrm{MeV}$ reflect the energy dependence of the low-energy neutron cross sections. The calculated and measured neutron spectra around $100 \mathrm{MeV}$ agree very well. The decrease of the measured spectral fluence rate above $200 \mathrm{MeV}$ is certainly too steep and is caused partly by the truncation of the spectrometer response matrix at $1 \mathrm{GeV}$.

In addition, we plot in Fig.7a the spectrum obtained from the LUIN-code [13,41]. LUIN is based on an essentially analytical theory of atmospheric cosmic-ray propagation. The validity of this approach is limited at low energies to about $100 \mathrm{MeV}$. In order to approximate the spectral neutron fluence rate below this threshold a measured spectrum [39] has been patched onto the calculated differential spectrum. It is exactly this measured spectrum which is shown in Fig.7a, normalized to the calculated value at $100 \mathrm{MeV}$. In spite of this fact it might be worthwhile to compare its shape with the two other spectra. We note, that the slope at low energies $(E<50 \mathrm{keV})$ is about equal to the one obtained from FLUKA. The evaporation "peak" is clearly pronounced, but in contrast to FLUKA and the measurements of Schraube et al. $[2,3]$ no enhancement at around $100 \mathrm{MeV}$ is seen.

We note, that the influence of the experimental setup and of the surroundings (such as the floor, snow etc.) on the neutron spectrum has neither been subtracted from the measured counting rates nor it was taken into consideration within the calculations. The same applies to the influence of atmospheric humidity. We assume that they may partly account for the differences between the unfolded and the calculated spectra at low energies. In order to study the influence of the surroundings on the neutron spectrum we consider as an extreme case a floor consisting of water with a standard density of $1 \mathrm{~g} / \mathrm{cm}^{3}$. In Fig. $7 \mathrm{~b}$ we compare our previously discussed spectrum with a neutron spectrum calculated behind $700 \mathrm{~g} / \mathrm{cm}^{2}$ air at the boundary between air and water. The only difference between the two calculations is that for the second calculation the lower air layers $\left(X_{v}>700 \mathrm{~g} / \mathrm{cm}^{2}\right)$ have been replaced by water; all other conditions are identical. As it can be seen, the influence of the surroundings on the neutron spectrum below $10 \mathrm{MeV}$ might be considerable.

A more detailed comparison with the spectra measured by Hess et al. [39], now in its normalization as measured, is presented in Fig.8. We show the neutron spectra for three different depths, $200 \mathrm{~g} / \mathrm{cm}^{2}, 700 \mathrm{~g} / \mathrm{cm}^{2}$, and $1030 \mathrm{~g} / \mathrm{cm}^{2}$. At the two higher depths the agreement between our calculations and the experimental data is satisfactorily, apart from a more pronounced neutron enhancement around $1 \mathrm{MeV}$ and a missing one around $100 \mathrm{MeV}$ within the Hess-data. At $200 \mathrm{~g} / \mathrm{cm}^{2}$ the experimental data below $10 \mathrm{MeV}$ are underestimated by the calculations. In order to investigate to which extend the previously shown spectrum by Schraube et al. is consistent with these measurements, we show it additionally in Fig. 8 . 
The experimental conditions concerning the primary cosmic ray flux and the geomagnetic shielding are comparable. Both measurements at $700 \mathrm{~g} / \mathrm{cm}^{2}$ differ in most of the energy range by a factor of two to ten (at $1 \mathrm{MeV}$ ), the spectral fluence rate of the Zugspitze-experiment being consistently lower below $10 \mathrm{MeV}$.

In Fig.9a the differential spectral neutron fluence rate for $40^{\circ} \mathrm{N} 110^{\circ} \mathrm{W}$, Oct. 1974 , and $200 \mathrm{~g} / \mathrm{cm}^{2}$ is compared to a corresponding measurement [40]. In difference to the two previously discussed experiments these data show neither the evaporation neutron source nor the enhancement at $100 \mathrm{MeV}$. However, below $100 \mathrm{keV}$ our calculations agree very well with the data.

Finally, in Fig.9b our calculations are plotted together with spectra measured by Nakamura et al. [42] at $200 \mathrm{~g} / \mathrm{cm}^{2}, 550 \mathrm{~g} / \mathrm{cm}^{2}$, and $1030 \mathrm{~g} / \mathrm{cm}^{2}$. Below $100 \mathrm{keV}$ our calculated spectra are consistently higher than the measurements. The evaporation spectra agree at $200 \mathrm{~g} / \mathrm{cm}^{2}$ and $550 \mathrm{~g} / \mathrm{cm}^{2}$. At higher energies, especially at sea level, the experimental uncertainties are too high to draw any conclusions [42].

\subsection{Depth dependence of neutron fluence rates}

An experimental investigation of the altitude and latitude dependence of the neutron fluence rate between 1 and $10 \mathrm{MeV}$ was performed during the solar minimum in 1964/65 by Holt et al. [43]. It should be kept in mind that this energy-range is rather small as compared to the range, for instance, the Zugspitze experiment was sensitive to and it covers only about half of the "evaporation peak". The experimental data are plotted in Fig.10 by polygonals. The uppermost curve (labelled position 1) corresponds to a location of $58.4^{\circ} \mathrm{N} 94.0^{\circ} \mathrm{W}$, i.e. a location with a rather weak magnetic shielding against low energy primaries $\left(E_{\text {prim }}>30 \mathrm{MeV}\right)$. In contrast, the bottom curve (labelled position 4) represents the neutron fluence rate measured at $17.2^{\circ} \mathrm{N} 78.3^{\circ} \mathrm{E}$, a location close to the geomagnetic equator. The two curves in between show measurements at intermediate positions (positions 2 and 3). Our calculations, performed for the same geographical locations and period of time, are given as filled dots. The agreement is reasonable in the two extreme cases, position 1 and 4 . The FLUKA-results nicely reproduce the rise of the slope with increasing latitude. In positions 2 and 3 FLUKA predicts significantly lower fluence rates at depths below $200 \mathrm{~g} / \mathrm{cm}^{2}$, whereas our results approach the experimental values at higher depths.

In order to understand the differences and the uncertainties inherent to the data we plot in addition an earlier measurement by the same group [44] (small open dots) for a geographical location comparable to position 2 . We note, that this measurement shows even higher fluence rates at low depths and a much stronger depth-dependence (bigger slope) than the later measurement. With respect to this observation it is difficult to decide if FLUKA is underestimating the atmospheric cascade at low depths or not.

In addition, the fluence rate of neutrons between 1 and $10 \mathrm{MeV}$ obtained from the unfolded spectrum at $700 \mathrm{~g} / \mathrm{cm}^{2}$ (Zugspitze) is shown. The strength of the geomagnetic shielding is about equal to the one in position 3 but the fluence rate is a factor of two lower than the calculated fluence rate. The latter might be in agreement with the extrapolation of the results of Holt et al. down to $700 \mathrm{~g} / \mathrm{cm}^{2}$. 


\subsection{The high energy behaviour of hadron spectra}

When measuring the vertical component of the high energy spectral hadron radiance ${ }^{4}$ at sea level one is typically triggering on primary particles which have undergone only a few interactions. Especially diffractive interactions are involved. Due to the close relation between primary protons and the vertical secondary hadrons which are selected, the slope of the vertical spectral hadron radiance should be similar to the slope in the primary spectrum. The single spectral hadron radiance from the vertical direction was measured at sea level with the KASKADE-experiment $[46,47]$. In Fig.11 we compare FLUKA-results on vertical spectral hadron and neutron radiances with these data. The ratio of accompanied neutrons to all neutrons was found to be lower than 0.1 within most of the energy range [47]. Therefore, we neglect the requirement of counting only unaccompanied hadrons in our calculations. We define "vertical hadrons" as those hadrons arriving at sea level with a zenith angle below $15^{\circ}$. A more detailed comparison would require to treat the geometry of the detector as it was done for the hadron reconstruction [46]. However, we found the calculated spectra to depend only weakly on the exact definition of the vertical direction. To speed up the calculations we imposed a lower cutoff in energy of $2 \mathrm{GeV}$ to all hadrons followed in the cascade. Primary protons were sampled up to an energy of $7 \mathrm{TeV}$. Our results agree with the measured spectral radiance within the statistical uncertainties of the calculations.

The vertical spectral radiance of protons with energies between $30 \mathrm{MeV}$ and $60 \mathrm{GeV}$ was measured at an altitude of $3200 \mathrm{~m}$ [48]. In Fig.12a we compare it with a corresponding calculation. Again, we take only protons with zenith angles below $15^{\circ}$ into consideration. The calculations have been performed with primary particles having energies between the lower cut of about $7 \mathrm{GeV}$ and $1.3 \mathrm{TeV}$. Secondaries were followed in the cascade down to $50 \mathrm{MeV}$. The agreement with the measurements is reasonable.

Finally, in Fig.12b we show the proton spectrum at an altitude of $5200 \mathrm{~m}$. It is compared to results of a measurement which was performed at Mt. Chacaltaya (Bolivia) [49]. Since it is not obvious to us to which zenith angles the experimental setup was sensitive we give our results for protons having zenith angles below $15^{\circ}$. In our transport calculations we have applied a lower cutoff in momentum of $200 \mathrm{MeV} / \mathrm{c}$.

\section{Summary and conclusions}

In the present work radiation fields in the atmosphere have been studied applying the FLUKA Monte Carlo model.

As a first step we have focused on the description of the primary cosmic ray spectra. It is discussed that the uncertainties within the measurements of the primary cosmic ray spectra may cause uncertainties in the estimation of secondary particle spectra in the atmosphere of the same order. We have shown that predictions for secondary hadron-, especially neutron-,

\footnotetext{
${ }^{4}$ Throughout this section we continue adopting the notation as suggested in Ref. [45]. In particular, we call the number of particles of a given kind, $d N$, incident upon an element of area, $d A$, per unit time, $d t$, within an element of solid angle, $d \Omega$, perpendicular to $d A$, and per unit energy, $d E$, "spectral radiance". We note however, that within experimental and theoretical studies of high energy hadron spectra this quantity is usually called "flux"
} 
spectra depend strongly on the lower energy-cutoff of the primary particle flux, which arises from geomagnetical shielding, and on the level of solar modulation. Therefore, we propose a model for the primary cosmic ray flux which is based on fits to data for solar minimum and maximum, on neutron monitor counting rates and which takes the rigidity-cutoff depending on the geomagnetical location and the direction of the primary into account. In this way we obtain estimations of the energy spectra of primary cosmic ray particles at any time, any location and for any directions of the primary which can be easily adopted for the sampling procedure of primaries within a MC-transport calculation.

Based on these "input" spectra and together with a slab-model of the atmosphere hadron energy spectra have been calculated for different depths in the atmosphere and compared to experimental data. Concerning the measurements of differential low energy neutron spectra the situation is still contradictory and the experimental results for the spectra of neutrons of a certain energy and at a certain depth may differ by up to a factor of ten. At this stage it is therefore difficult to draw definite conclusions. With regard to the comparison with the Zugspitze experiment one can say that despite of all differences and taking into consideration the uncertainties both, within the measurements including the response calculations, and within the FLUKA calculations, an agreement is observed at least within parts of the energy range under investigation. However, a series of systematical measurements performed at different depths and latitudes seems to us highly desirable. The solar minimum during the years 1996/97 would provide relatively stable conditions of increased primary cosmic ray fluxes. As soon as data from future measurements are available further studies in comparing them to FLUKA predictions are necessary.

At higher energies the vertical spectral hadron radiance in the atmosphere is in agreement with recent measurements from the KASKADE experiment and with results from two other experiments performed at different geographical locations and different altitudes.

In order to reduce the considerable uncertainties entering our calculations with respect to the primary cosmic ray spectrum, updates of the fits to these spectra are needed as soon as more experimental information is obtained.

We conclude that FLUKA may serve as important tool for the estimation of the radiation environment in the atmosphere and behind shielding materials of aircrafts since particle spectra can be calculated for any position and time in the atmosphere. Its predictive power arises mainly from the fact that it proved to be very reliable in numerous applications at high energy accelerators where, in contrast to cosmic ray cascades, detailed comparisons with data on various aspects are possible.

\section{Acknowledgements}

We want to thank K. O'Brien, A. Ferrari, J. Ranft, and G. R. Stevenson for numerous stimulating discussions and A. Ferrari and J. Ranft for critical comments on the manuscript. We are grateful to A. Ferrari for providing the FLLKA-95 version of the code. This work was partially supported by the European Commission of the European Union DGXII unter contracts FI3P-CT92-0026 and CT95-0008. One of the authors (S.R.) was partially supported by a grant of the Bundesland Nordrhein-Westfalen. 


\section{References}

[1] H. G. Paretzke and W. Heinrich: Radiation Protection Dosimetry 48 (1993) 33

[2] H. Schraube, J. Jakes, A. Sannikov, E. Weitzenegger, S. Roesler and W. Heinrich: The cosmic ray induced neutron spectrum on top of the Zugspitze (2963m), Presented at the 8th Symposium on Neutron Dosimetry, Paris, France, 1995, accepted for publication in Radiation Protection Dosimetry, 1996

[3] H. Schraube, G. Leuthold, S. Roesler and W. Heinrich: Neutron spectra at flight altitudes and their radiological estimation, Presented at the 31st COSPAR Scientific Assembly, Birmingham, U.K., 1996, to be published in Advances in Space Research , 1996

[4] C. Birattari et al.: Measurements and Simulations in High Energy Neutron Fields, CERN/TIS-RP/96-5/CF, presented at the 2nd Specialists' Meeting on Shielding Aspects of Accelerators, Targets and Irradiation Facilities, CERN, Geneva, Switzerland, 1995

[5] G. Battistoni, C. Forti and J. Ranft: Astroparticle Phys. 3 (1995) 157

[6] G. Battistoni, et al.: Calculation of the TeV Prompt Muon Component in Very High Energy Cosmic Ray Showers, LNF-95-038-P, 1995

[7] G. Battistoni: Monte Carlo simulations in high-energy cosmic ray physics, Presented at the IVth International Conference on Calorimetry in High Energy Physics, La Biodola (Elba), Italy, 1993, Published in Calorimetry in HEP 1993, p. 503, 1993

[8] V. Patera, M. Carboni, G. Battistoni and A. Ferrari: Nucl. Instrum. Methods A356 (1995) 514

[9] C. Forti et al.: Phys. Rev. D42 (1990) 3668

[10] R. S. Fletcher, T. K. Gaisser, P. Lipari and T. Stanev: Phys. Rev. D50 (1994) 5710

[11] T. W. Armstrong, K. C. Chandler and J. Barish: J. Geophys. Res. 78 (1973) 2715

[12] M. Merker: Health Physics 25 (1973) 524

[13] K. O'Brien: Il Nuovo Cimento 3A (1971) 521

[14] K. O'Brien: J. Phys. A: Math. Gen. 8 (1975) 1530

[15] C. Pfeifer, S. Roesler and M. Simon: Phys. Rev. C54 (1996) 882

[16] A. Fassò, A. Ferrari, J. Ranft and P. R. Sala: FLUKA: present status and future developments, in Proceedings of the IVth International Conference on Calorimetry in High Energy Physics, La Biodola (Elba), Italy, 1993, ed. by A. Menzione and A. Scribano, World Scientific, p. 493, 1994 
[17] A. Fassò, A. Ferrari, J. Ranft and P. R. Sala: FLUKA: Performances and Applications in the Intermediate Energy Range, in Proceedings of the 1st Specialists' Meeting on Shielding Aspects of Accelerators, Targets and Irradiation Facilities, Arlington, U.S.A., 1994, published by OECD/NEA, p. 287, 1994

[18] P. A. Aarnio et al.: in Proceedings of the International Conference on Monte Carlo Simulation in High Energy and Nuclear Physics, MC'93, Tallahassee, U.S.A., 1993, ed. by P. Dragovitsch, S. L. Linn and M. Burbank, World Scientific, p. 88, 1994

[19] L. Waters et al.: The current status of LAHET/MCNP, presented at the 2nd Workshop on Simulating Accelerator Radiation Environments, CERN, Geneva, Switzerland, 1995

[20] N. Yoshizawa et al.: Development of high energy transport code HETC-3STEP, presented at the 2nd Workshop on Simulating Accelerator Radiation Environments, CERN, Geneva, Switzerland, 1995

[21] M. Höfert and G. R. Stevenson: The CERN-CEC High-Energy Reference Field Facility, CERN/TIS-RP/94-2/CF, presented at the American Nuclear Society's 8th International Conference on Radiation Shielding, Arlington, U.S.A., 1994

[22] A. Capella, U. Sukhatme, C. I. Tan and J. Trân Thanh Vân: Phys. Rep. 236 (1994) 227

[23] K. Hänssgen and J. Ranft: Comp. Phys. Commun. 39 (1986) 37

[24] P. R. Sala and A. Ferrari: A new model for hadronic interactions at intermediate energies for the FLUKA code, in Proceedings of the International Conference on Monte Carlo Simulation in High Energy and Nuclear Physics, MC'93, Tallahassee, U.S.A., 1993, ed. by P. Dragovitsch, S. L. Linn and M. Burbank, World Scientific, p. 277, 1994

[25] A. Ferrari, J. Ranft, S. Roesler and P. R. Sala: Z. Phys. C70 (1996) 413

[26] A. Ferrari, J. Ranft, S. Roesler and P. R. Sala: Z. Phys. C71 (1996) 75

[27] A. Ferrari and P. R. Sala: Physics of Showers induced by Accelerator Beams, Lecture given at the 1995 "Frederic Joliot" Summer School in Reactor Physics, Cadarache, France, 1995

[28] A. Ferrari, P. R. Sala, R. Guaraldi and F. Padoani: Nucl. Instrum. Methods B71 (1992) 412

[29] E. Cuccoli, A. Ferrari and G. C. Panini: A group library from JEF 1.1 for flux calculations in the LHC machine detectors, ENEA-Bologna Report JEF-DOC-340, 1991

[30] A. Ferrari: private communication,

[31] T. K. Gaisser: Cosmic Rays and Particle Physics Cambridge University Press Cambridge 1990 
[32] J. H. Adams, Jr., R. Silberberg and C. H. Tsao: Cosmic Ray Effects on Microelectronics, Part I: The Near- Earth Particle Environment, NRL Memorandum Report 4506, 1981

[33] J. Engel, T. K. Gaisser, P. Lipari and T. Stanev: Phys. Rev. D46 (1992) 5013

[34] G. Battistoni, C. Forti, J. Ranft and S. Roesler: Deviations from the superposition model in a Dual Parton Model with formation zone cascade in both projectile and target nuclei, US-FT/29-96 (hep-ph/9606485), submitted for publication in Astroparticle Phys., 1996

[35] W. Heinrich and A. Spill: J. Geophys. Res. 84 (1979) 4401

[36] M. A. Shea, D. F. Smart and J. R. McCall: Can. J. Phys. 46 (1988) S1098

[37] M. Ichimura et al.: Phys. Rev. D48 (1993) 1949

[38] O. C. Allkofer and P. K. F. Grieder: Physics Data: Cosmic Rays on Earth Fachinformationszentrum Energie - Physik - Mathematik GmbH Karlsruhe 1984

[39] W. N. Hess, H. W. Patterson, R. Wallace and E. L. Chupp: Phys. Rev. 116 (1959) 445

[40] J. E. Hewitt et al.: Health Physics 34 (1978) 375

[41] K. O'Brien: LUIN: A code for the calculation of cosmic ray propagation in the atmosphere, US Department of Energy Report EML-338, (update of HASL-275), 1978

[42] T. Nakamura, Y. Uwamino, T. Ohkubo and A. Hara: Health Physics 53 (1987) 509

[43] S. S. Holt, R. B. Mendell and S. A. Korff: J. Geophys. Res. 71 (1966) 5109

[44] R. B. Mendell and S. A. Korff: J. Geophys. Res. 68 (1963) 5487

[45] ICRU-Report 33: Radiation Quantities and Units, International Commission on Radiation Units and Measurements, 1980

[46] H. H. Mielke, M. Föller, J. Engler and J. Knapp: J. Phys. G 20 (1994) 637

[47] H. Kormmayer, H. H. Mielke, J. Engler and J. Knapp: J. Phys. G 21 (1995) 439

[48] N. M. Kocharian, G. S. Saakian and Z. A. Kirakosian: Sov. Phys. JETP 35 (1959) 933

[49] O. C. Allkofer and E. Kraft: Il Nuovo Cimento XXXIX (1965) 1051 


\section{Figure Captions}

1. Dependence of the vertical height above sea level on the vertical depth according to a fit to the profile of the "standard atmosphere" [31]. The dots correspond to the slab structure entering the calculations.

2. In (a) the differential energy spectrum of primary cosmic ray nucleons in the absence of the geomagnetic field is shown for the two extreme cases, solar minimum and solar maximum, (dotted lines) and for May 1995 (full line). Stars represent the primary spectrum at the geographical position of the Zugspitze. Below $50 \mathrm{GeV}$ the curves are fits to experimental data [32] and above a power law spectrum [37] is patched. In (b) the normalized monthly average neutron rate measured by the neutron monitor at Deep River (Canada) is given for the years 1958-1995. The average counting rates per hour are obtained from the WWW-pages at the World Data Center (Boulder). The smooth curve is a sinusoidal fit to the data.

3. Omnidirectional neutron (a) and charged hadron (b) spectra as calculated with FLUKA are shown for three different depths in the atmosphere. The input spectrum of primary particles corresponds to the conditions in May 1995 and the geographical position of the Zugspitze.

4. FLUKA results for neutron $(E \geq 0.414 \mathrm{eV})$ and charged hadron fluence rates are given as function of the vertical atmospheric depth for May 1995. The lines represent fits to an exponential behaviour (solid line: $X_{v}>400 \mathrm{~g} / \mathrm{cm}^{2}$, dotted line: $X_{v}>600 \mathrm{~g} / \mathrm{cm}^{2}$ ).

5. The depth dependence of the neutron fluence rate is calculated for different input conditions: geomagnetic pole and solar minimum (filled dots), geomagnetic pole and solar maximum (stars), geomagnetic equator (open dots). The straight lines are fits to the results for $X_{v}>400 \mathrm{~g} / \mathrm{cm}^{2}$ and represent the exponential dependence of the fluence rate on the atmospheric depth.

6. The depth dependence of the charged hadron fluence rate is calculated for different input conditions (as in Fig.5).

7. In a) the experimentally determined spectral neutron fluence rate on top of the Zugspitze (2963m, reference depth: $\left.X_{v}=700 \mathrm{~g} / \mathrm{cm}^{2}\right)[2,3]$ (solid line) is compared to predictions of FLUKA (dots with error bars) and of LUIN $[13,41]$ (dotted line). In b) the calculated spectra at $700 \mathrm{~g} / \mathrm{cm}^{2}$ and at the geographical position of the Zugspitze over air (labelled "FLUKA") and over water ("FLUKA $\left(\mathrm{H}_{2} \mathrm{O}\right)$ ") are compared.

8. Differential energy spectra of neutrons at $200 \mathrm{~g} / \mathrm{cm}^{2}, 700 \mathrm{~g} / \mathrm{cm}^{2}$, and $1030 \mathrm{~g} / \mathrm{cm}^{2}$ (from the top to the bottom) calculated with FLUKA are shown together with measurements of Hess et al. [39] and Schraube et al. $[2,3]$.

9. Neutron energy spectra calculated with FLUKA for the conditions of two different experiments: Hewitt et al. [40] at $200 \mathrm{~g} / \mathrm{cm}^{2}$ (a) and Nakamura et al. [42] at $200 \mathrm{~g} / \mathrm{cm}^{2}$, $550 \mathrm{~g} / \mathrm{cm}^{2}$, and at sea level (b). 
10. Dependence of the neutron fluence rate between $1 \mathrm{MeV}$ and $10 \mathrm{MeV}$ on the atmospheric depth. FLUKA results (filled dots) are compared to measured fluence rates (lines) [43] for the solar minimum in 1964/65 and four different geographical locations; labelled with position 1 to 4 from top to the bottom: $58^{\circ} \mathrm{N} 94^{\circ} \mathrm{W}, 43^{\circ} \mathrm{N} 96^{\circ} \mathrm{W}, 31^{\circ} \mathrm{N} 95^{\circ} \mathrm{W}$, and $17^{\circ} \mathrm{N} 78^{\circ} \mathrm{E}$. In addition, an earlier measurement at a geographical location of $47^{\circ} \mathrm{N} 97^{\circ} \mathrm{W}$ (small open dots) [44] and the value obtained at the Zugspitze $\left(47^{\circ} \mathrm{N} 11^{\circ} \mathrm{E}, 700 \mathrm{~g} / \mathrm{cm}^{2}\right.$, big open dot) $[2,3]$ are plotted.

11. The calculated vertical spectral hadron (a) and neutron radiance (b) at sea level is shown together with measurements [47]. From the FLUKA calculations all hadrons at sea level with zenith angles below $15^{\circ}$ were counted.

12. The vertical spectral proton radiance at an altitude of $3200 \mathrm{~m}\left(700 \mathrm{~g} / \mathrm{cm}^{2}\right)$ is compared to measurements [48] (a). From the FLUKA calculations all protons at sea level with zenith angles below $15^{\circ}$ were counted. In (b) the proton spectrum at an altitude of $5200 \mathrm{~m}\left(535 \mathrm{~g} / \mathrm{cm}^{2}\right)$ in downward direction and with zenith angles below $15^{\circ}$ is shown together with measurements [49]. 


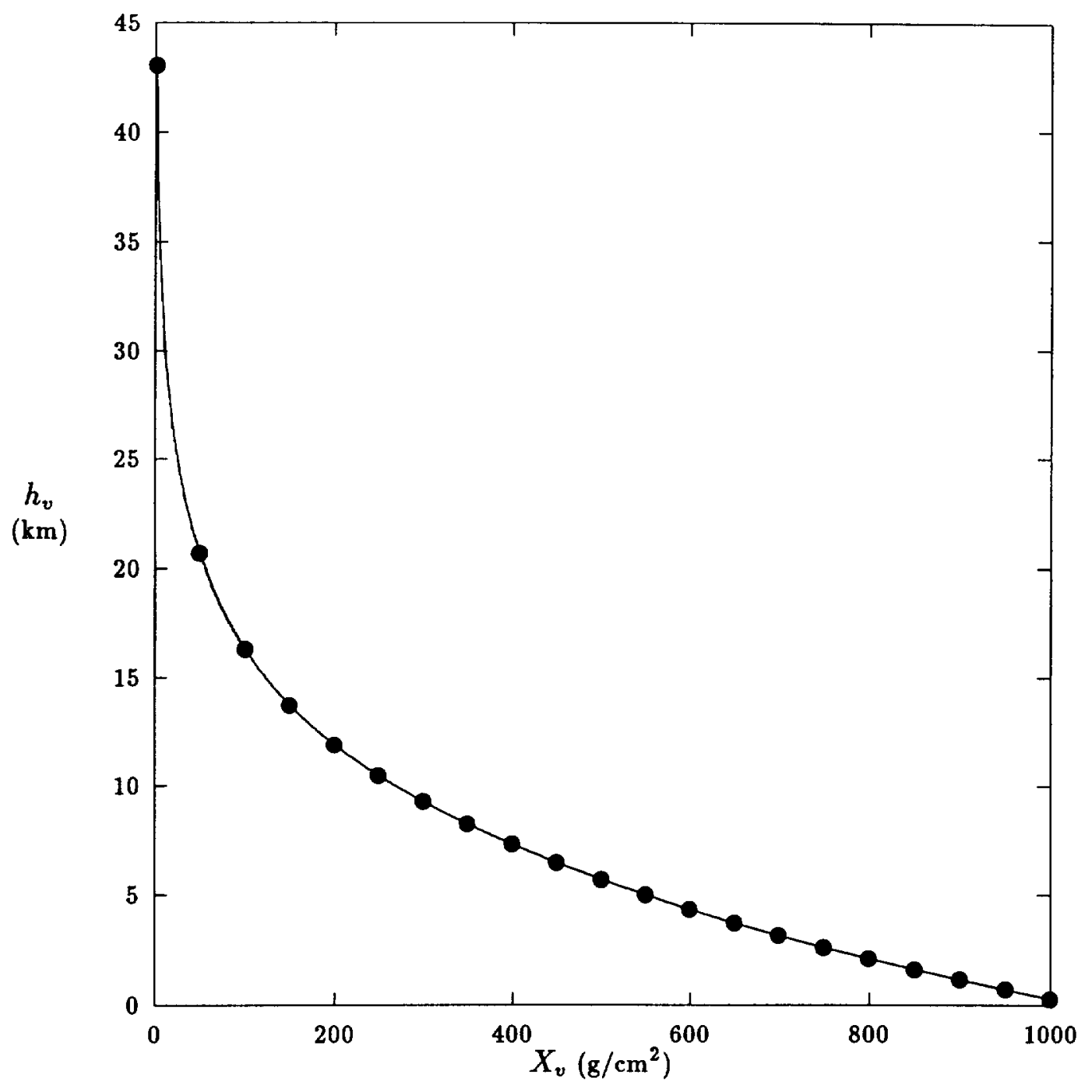

Fig. 1 


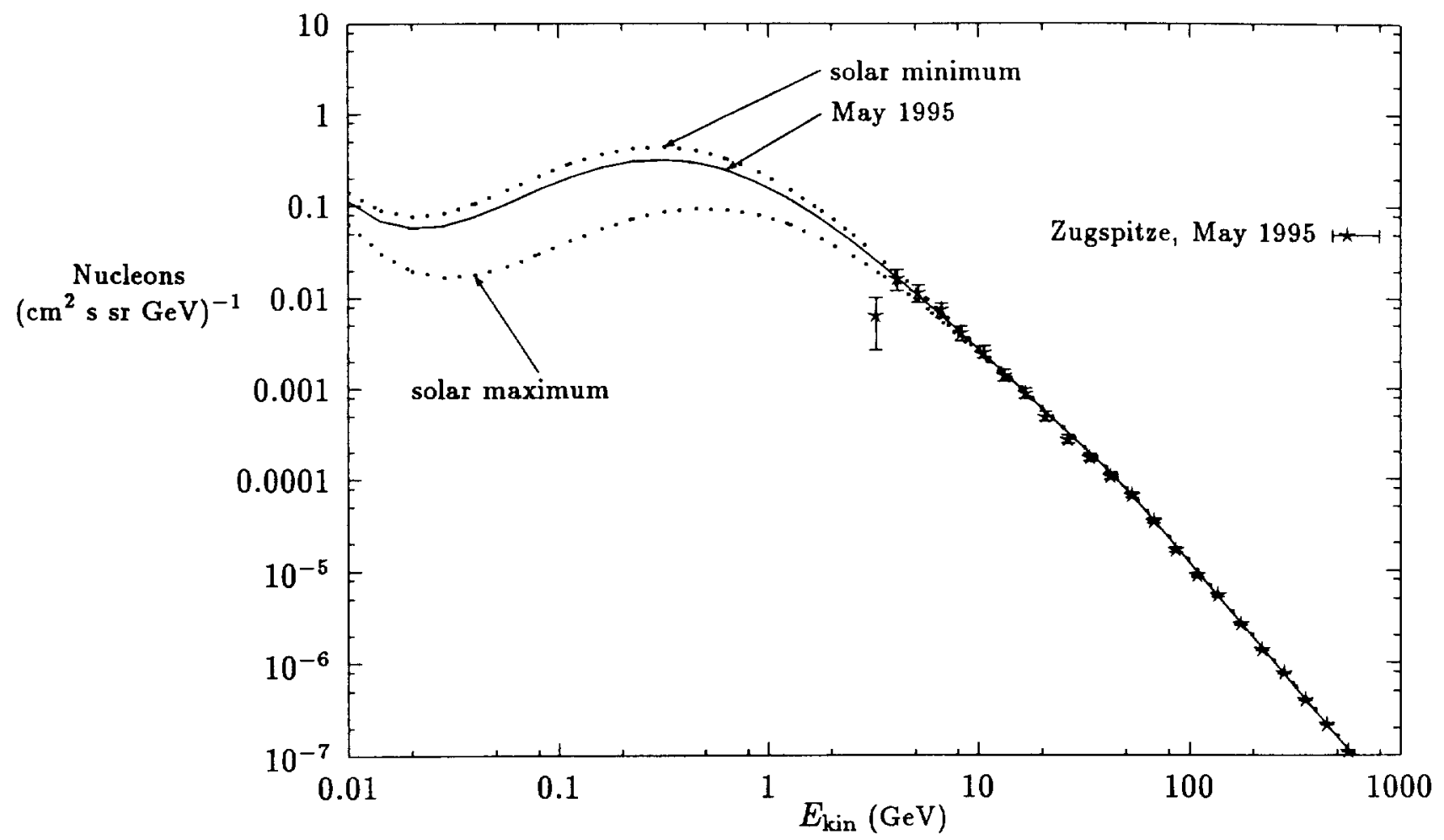

a)

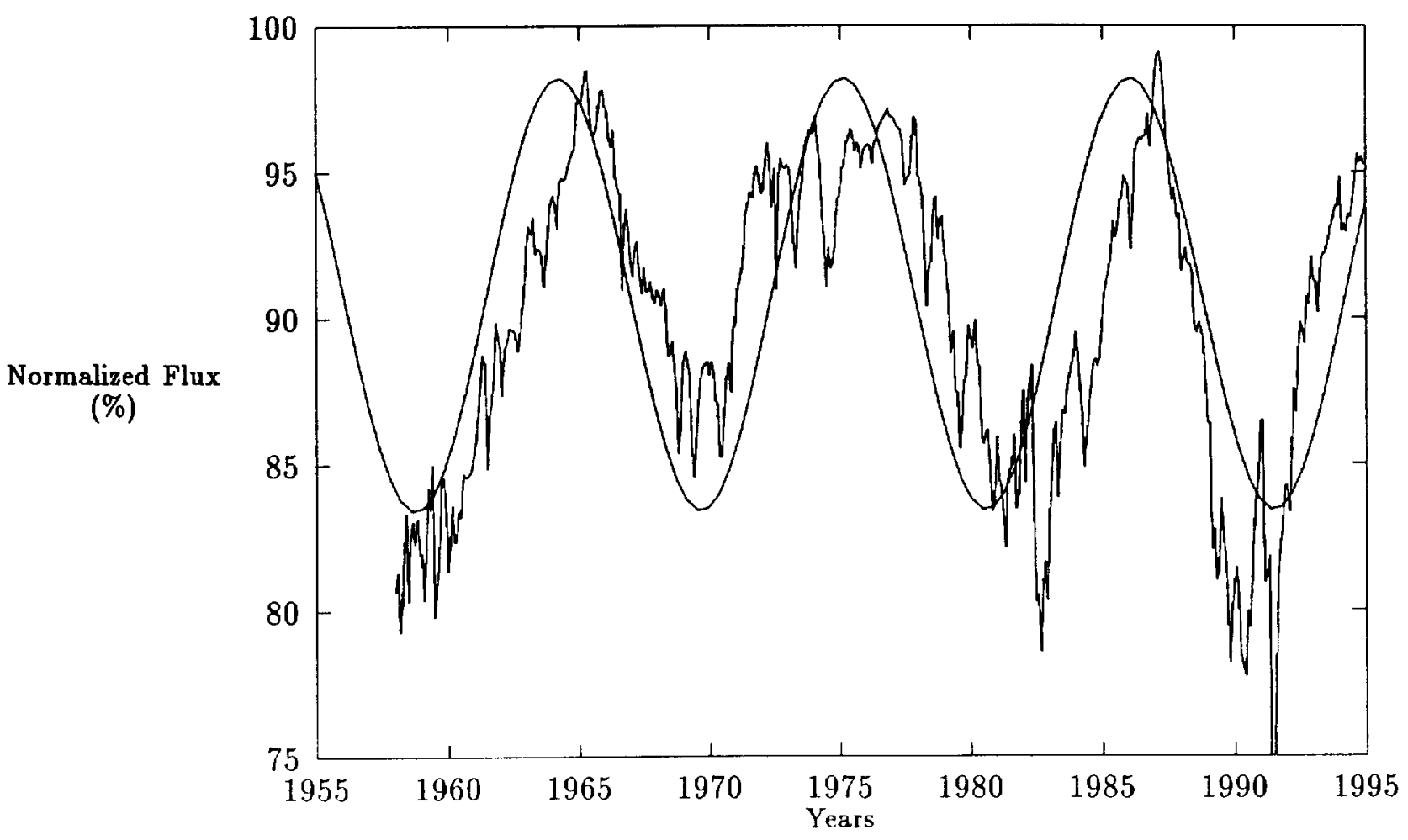

b)

Fig. 2 


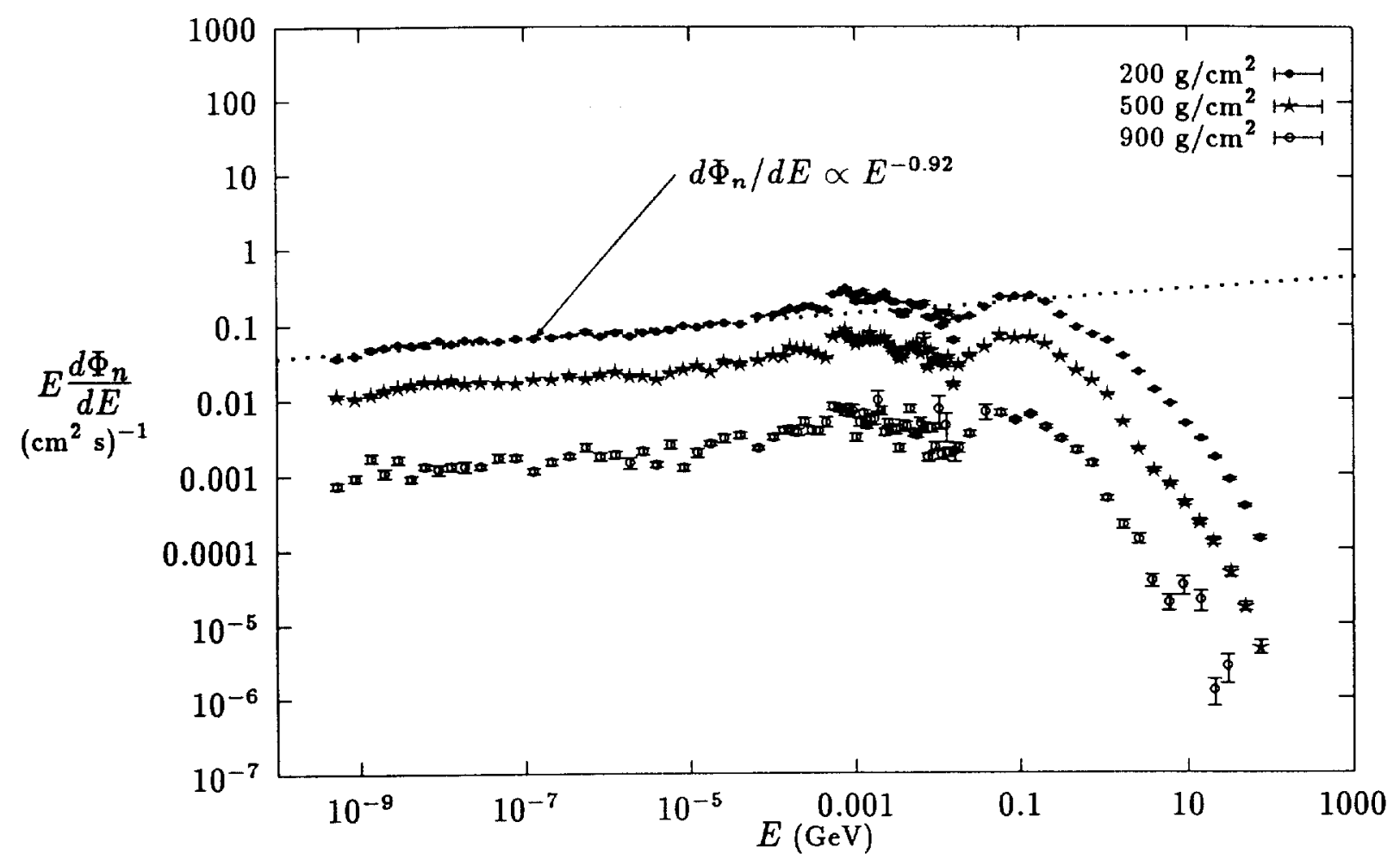

a)

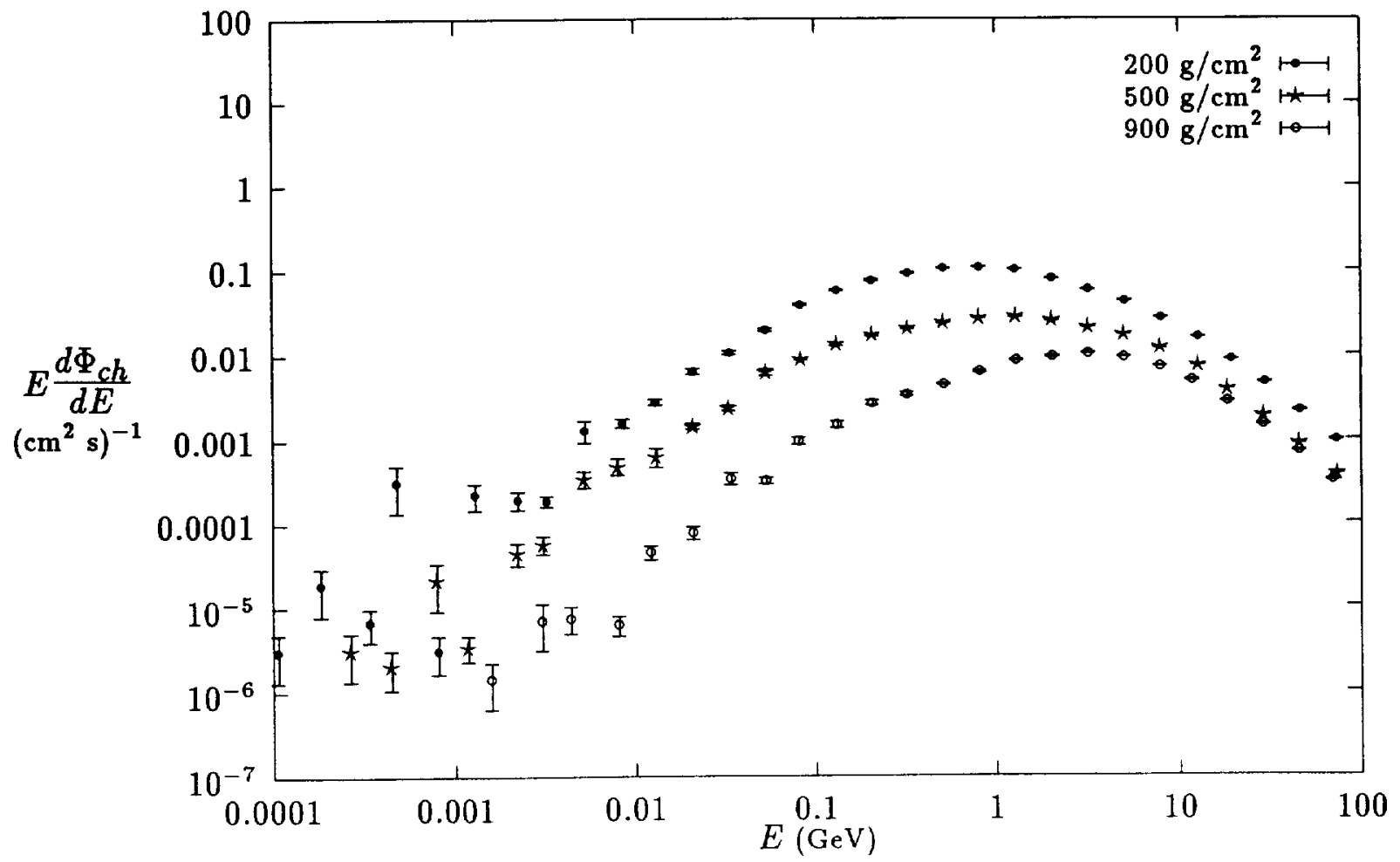

b)

Fig. 3 


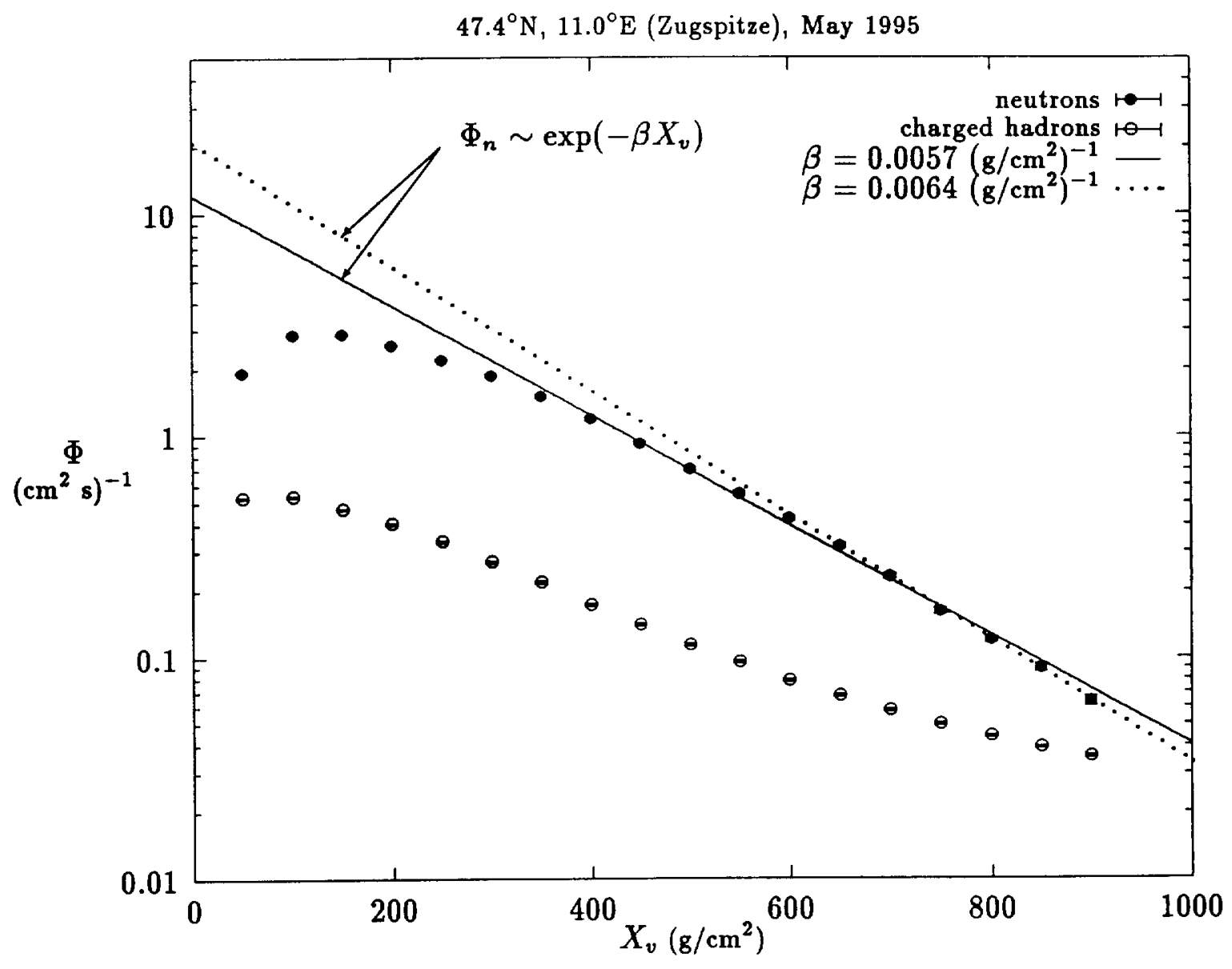

Fig. 4 


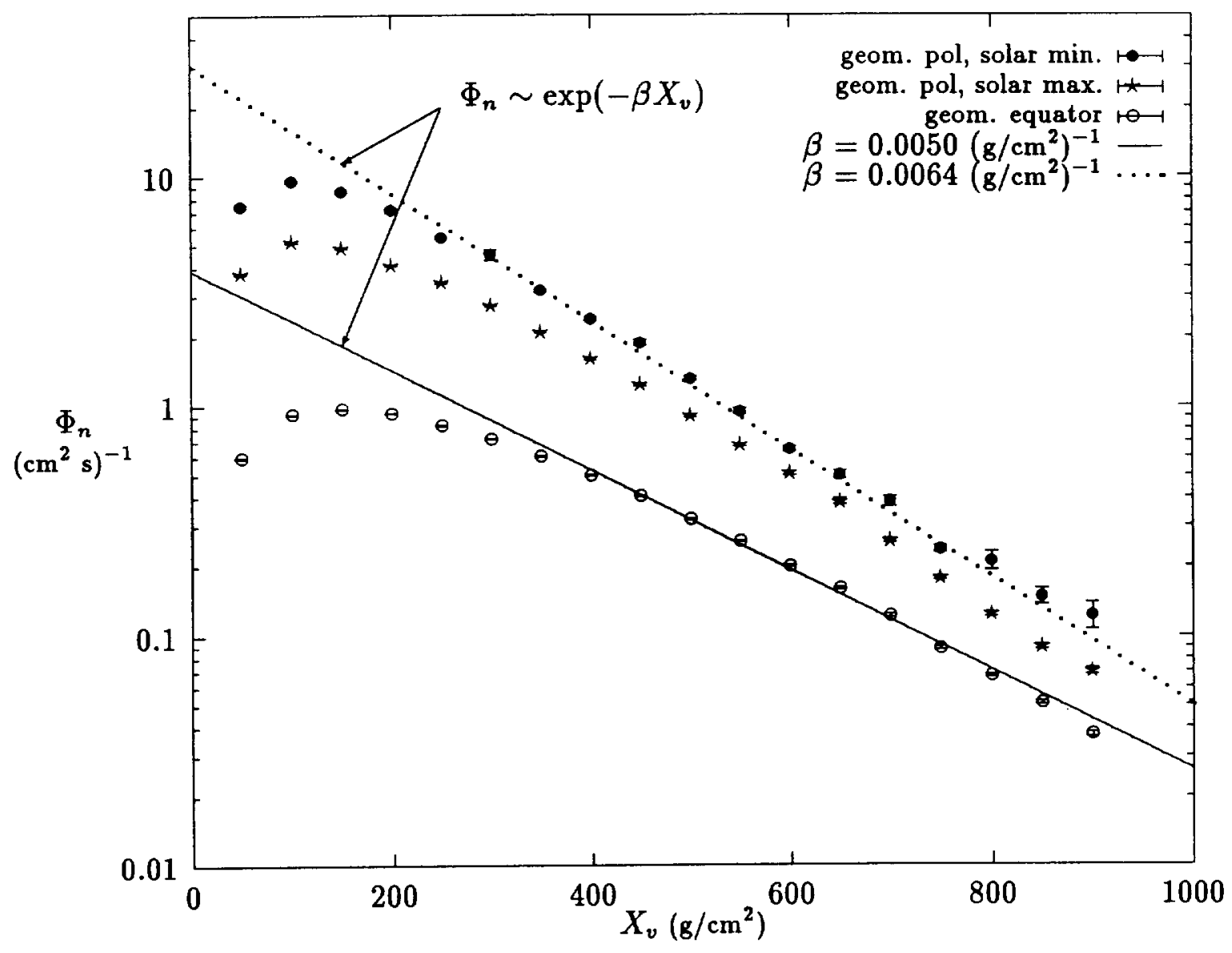

Fig. 5 


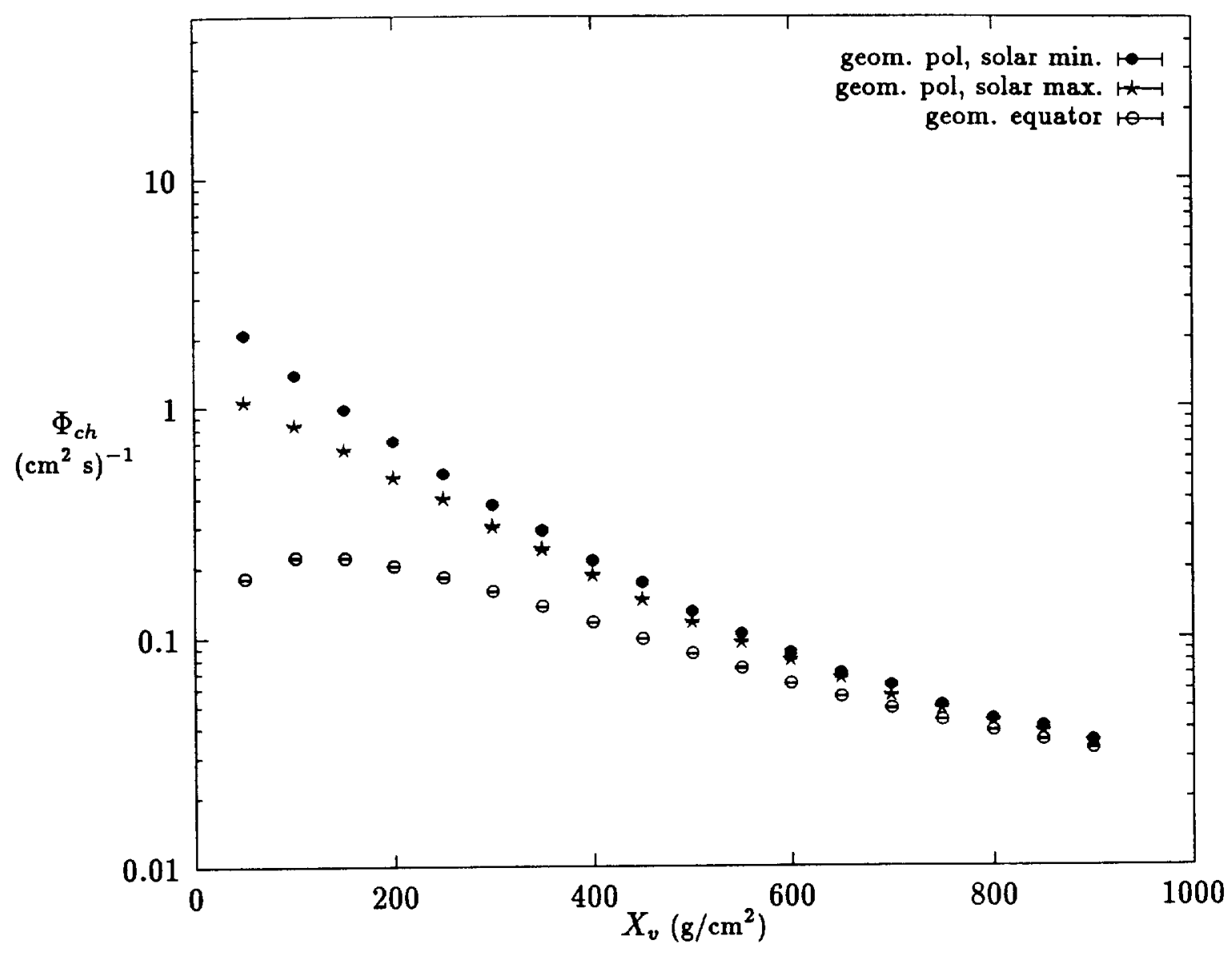

Fig. 6 


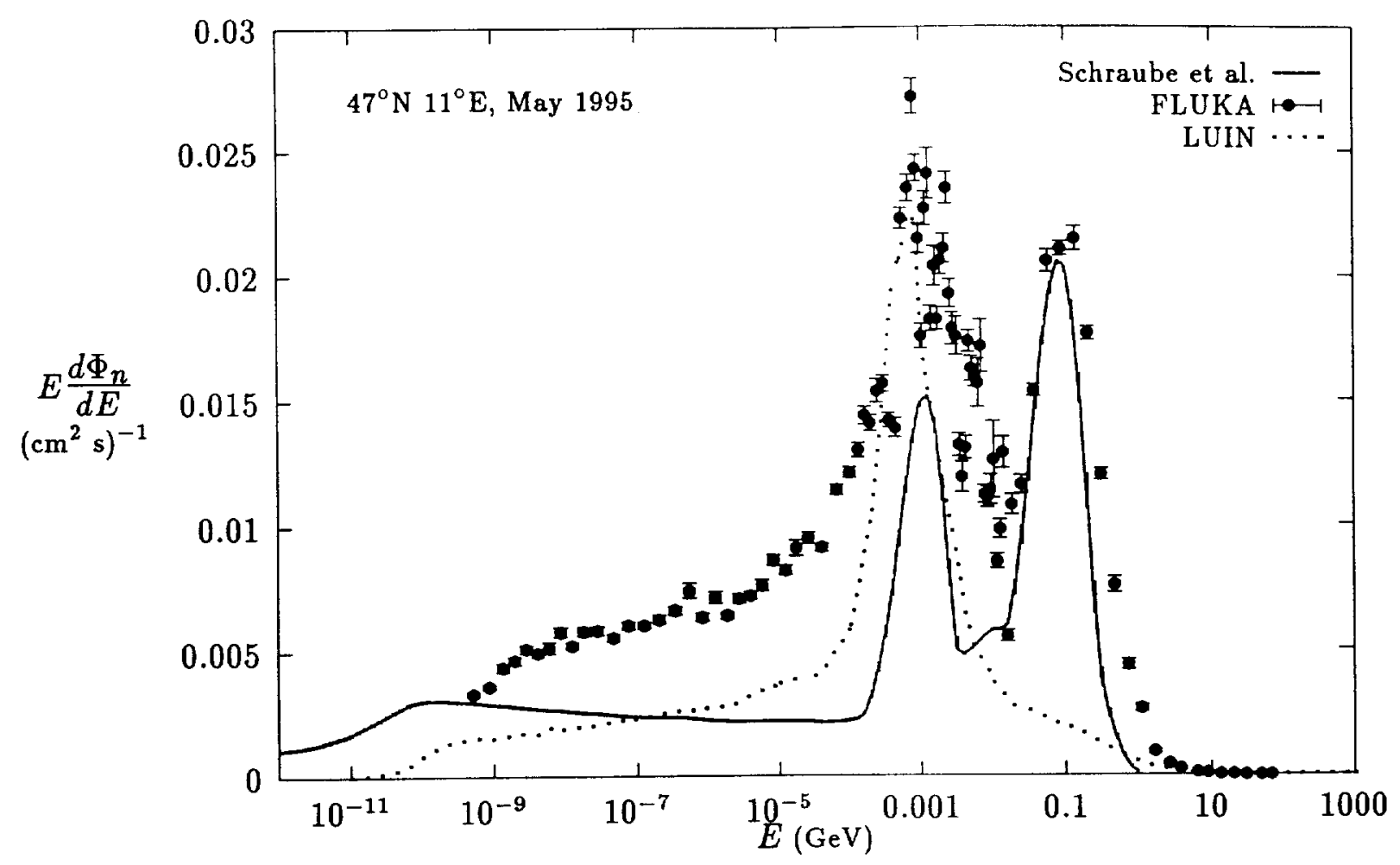

a)

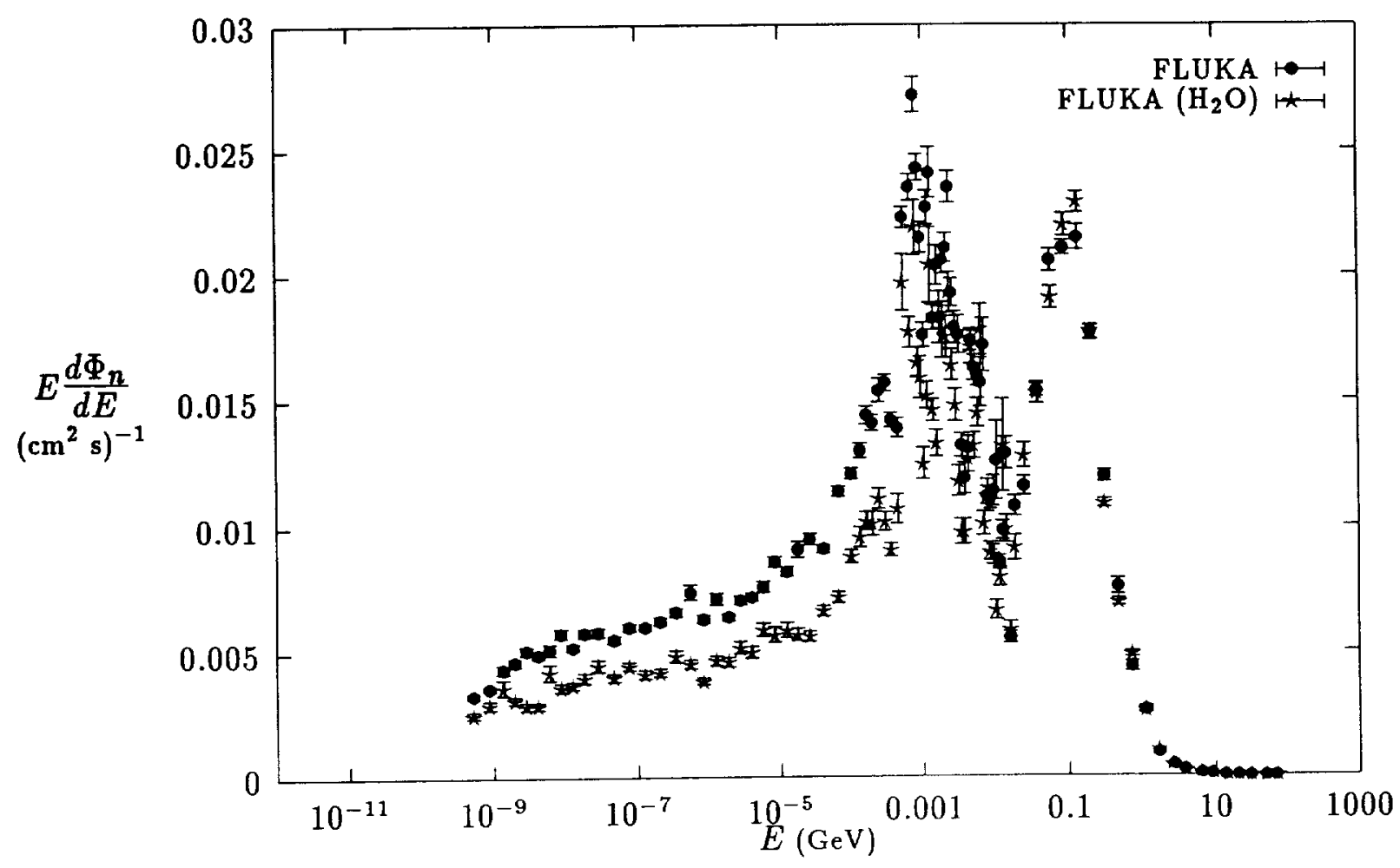

b)

Fig. 7 


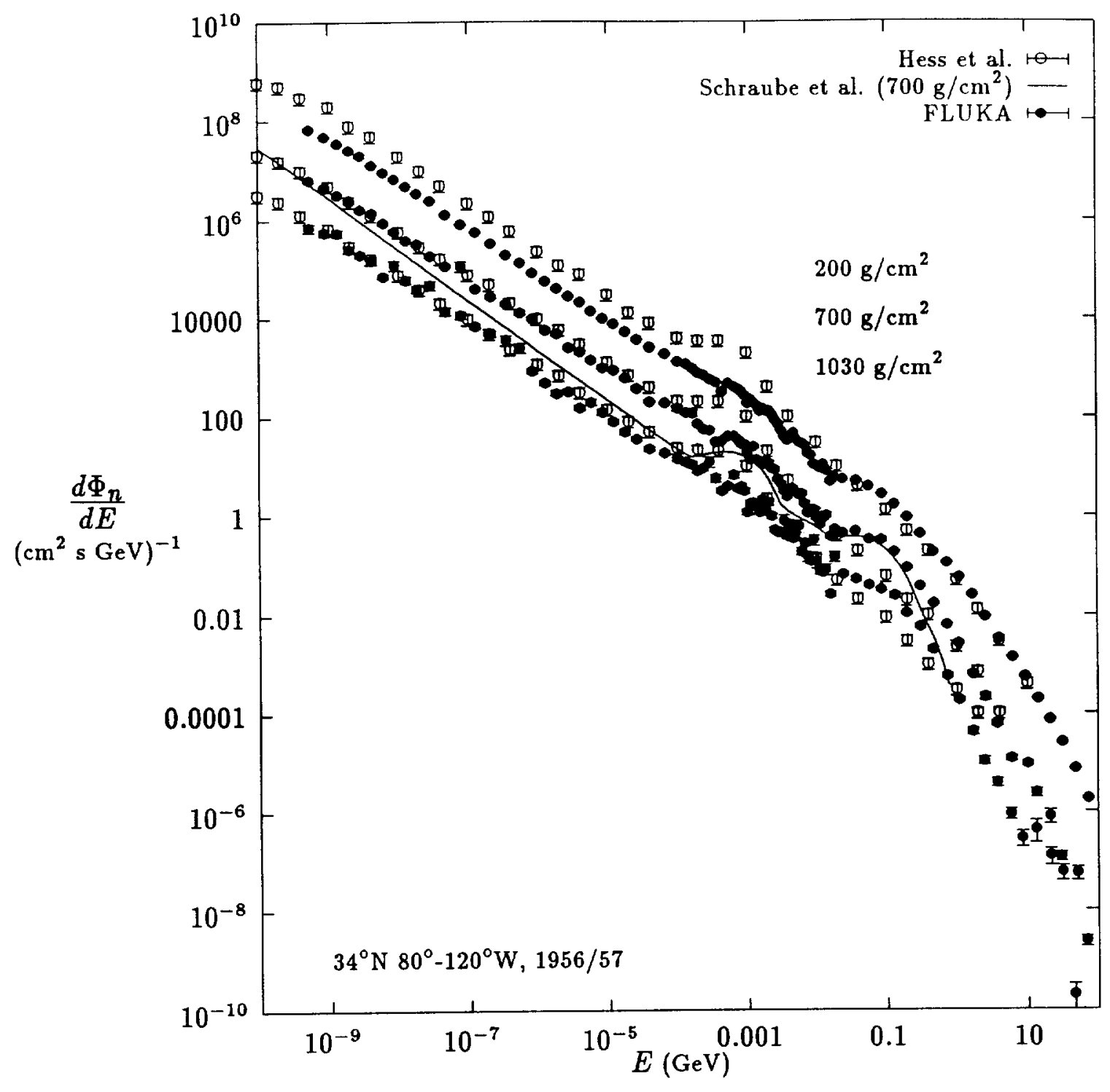

Fig. 8 


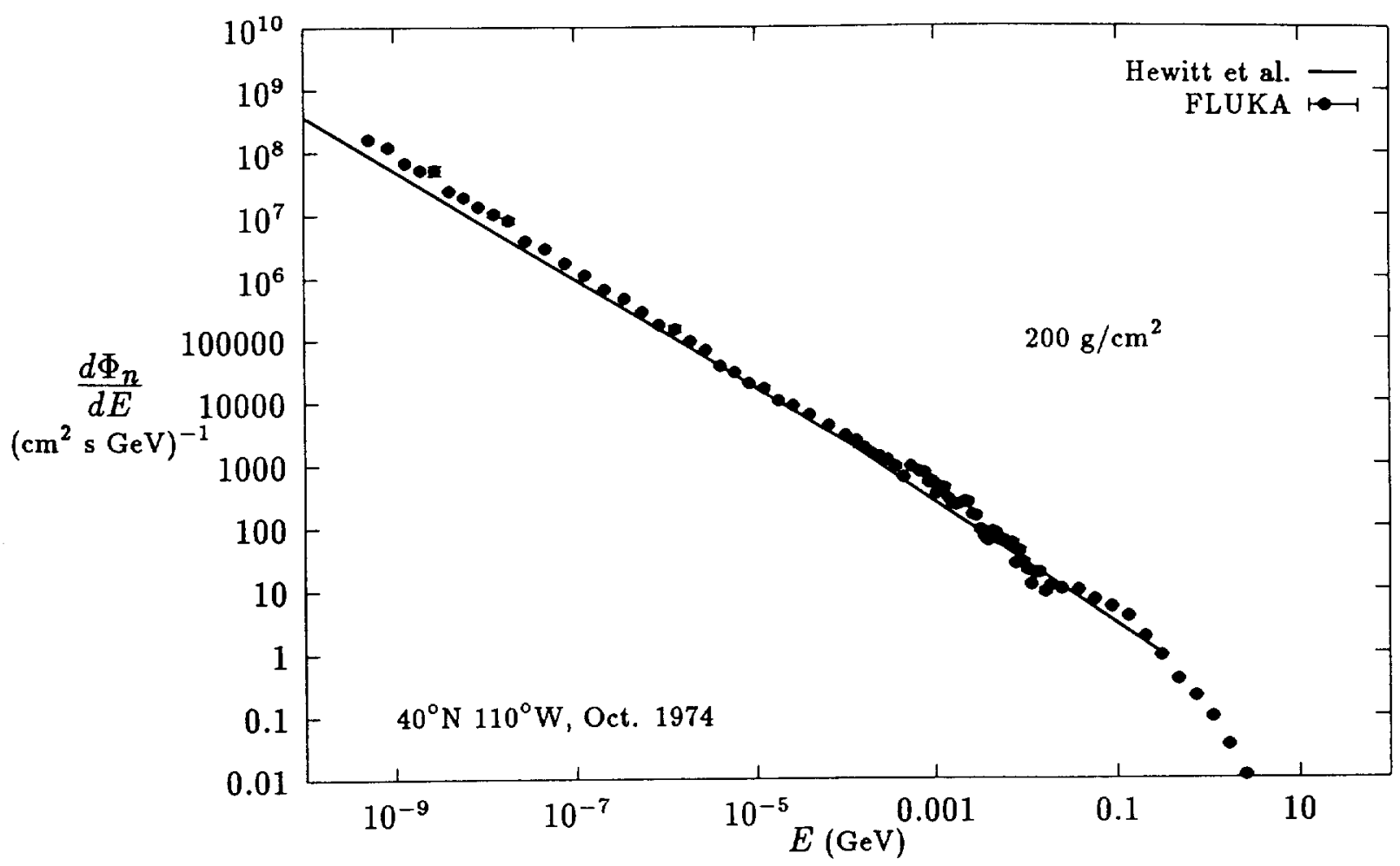

a)

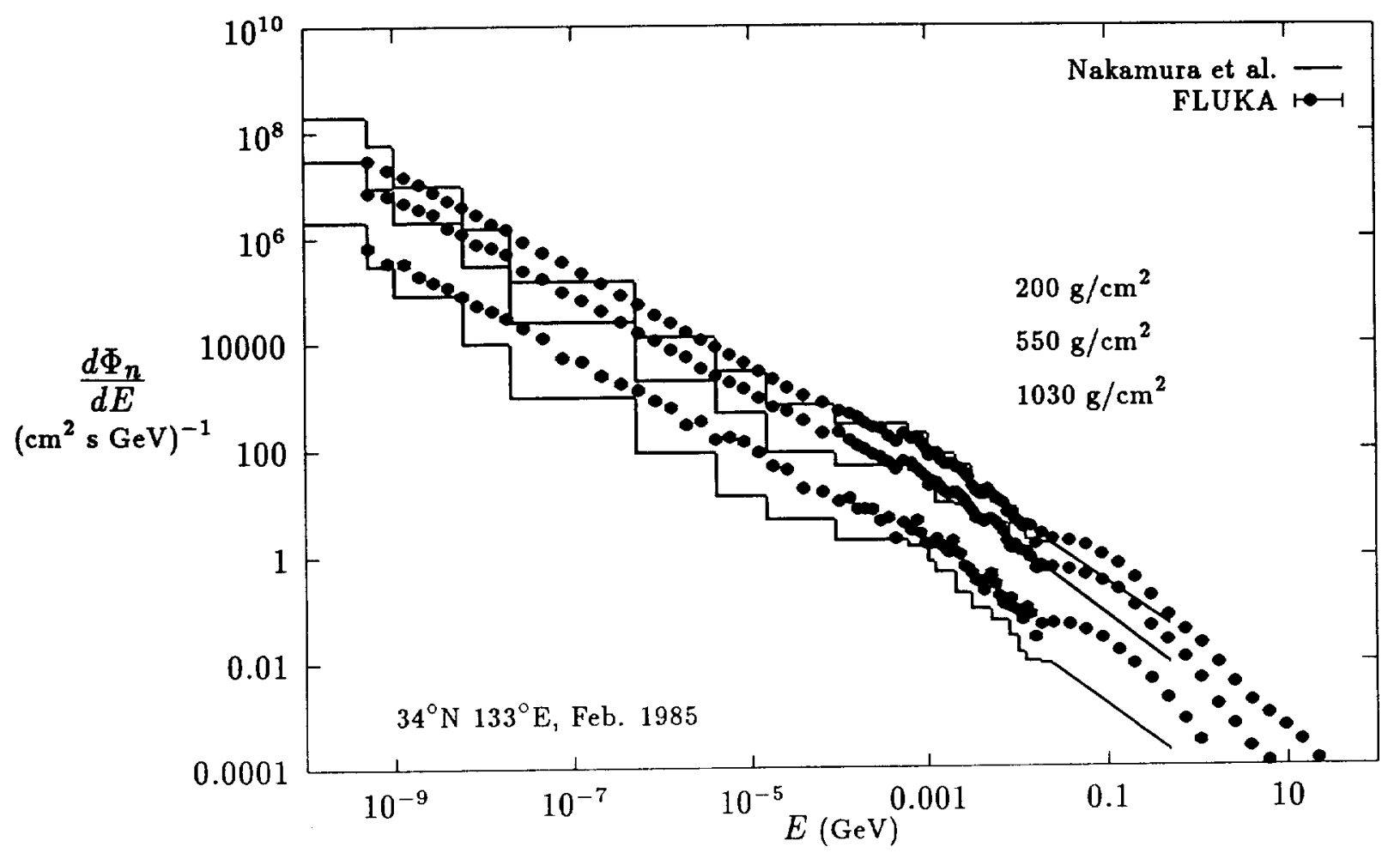

b)

Fig. 9 


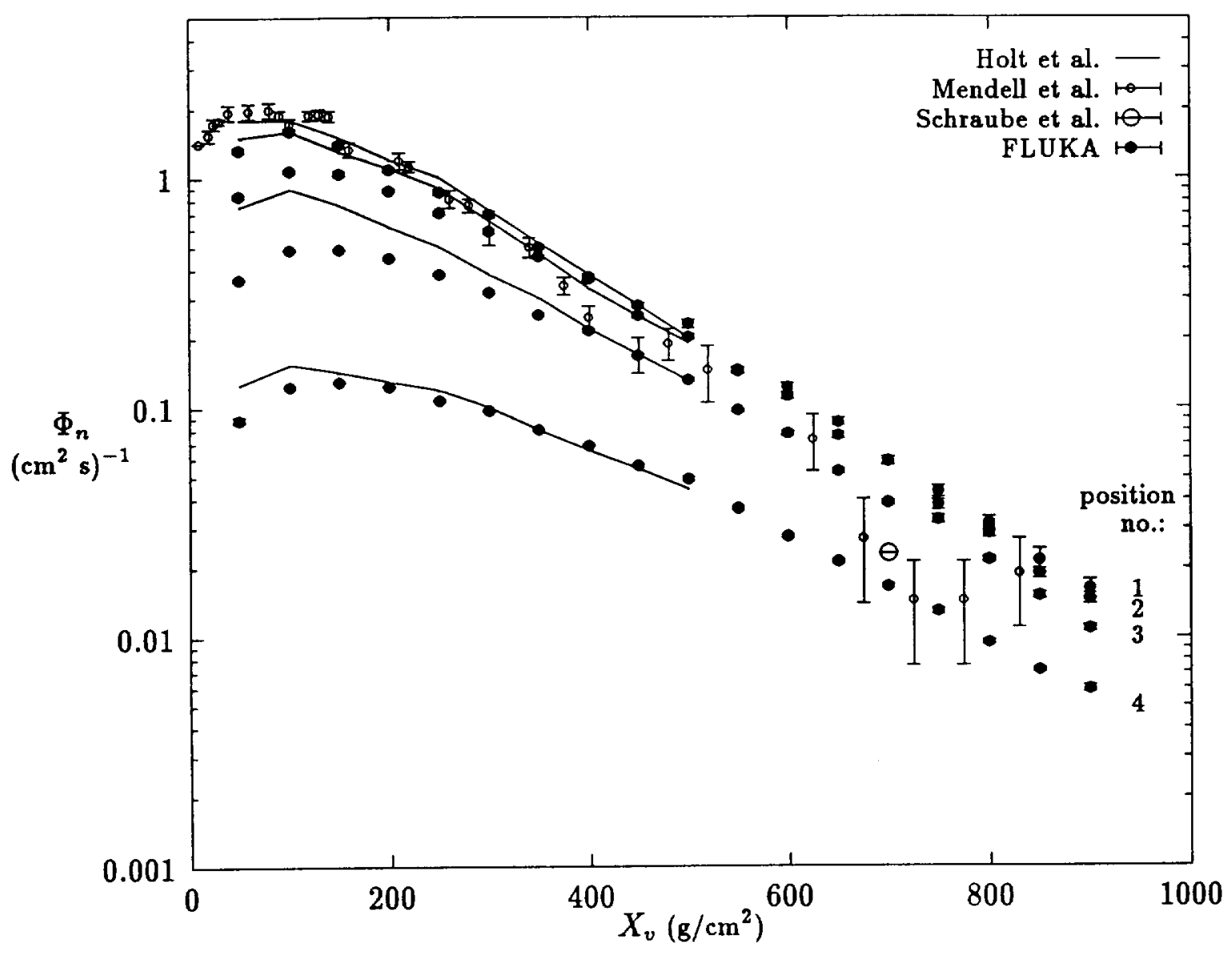

Fig. 10 


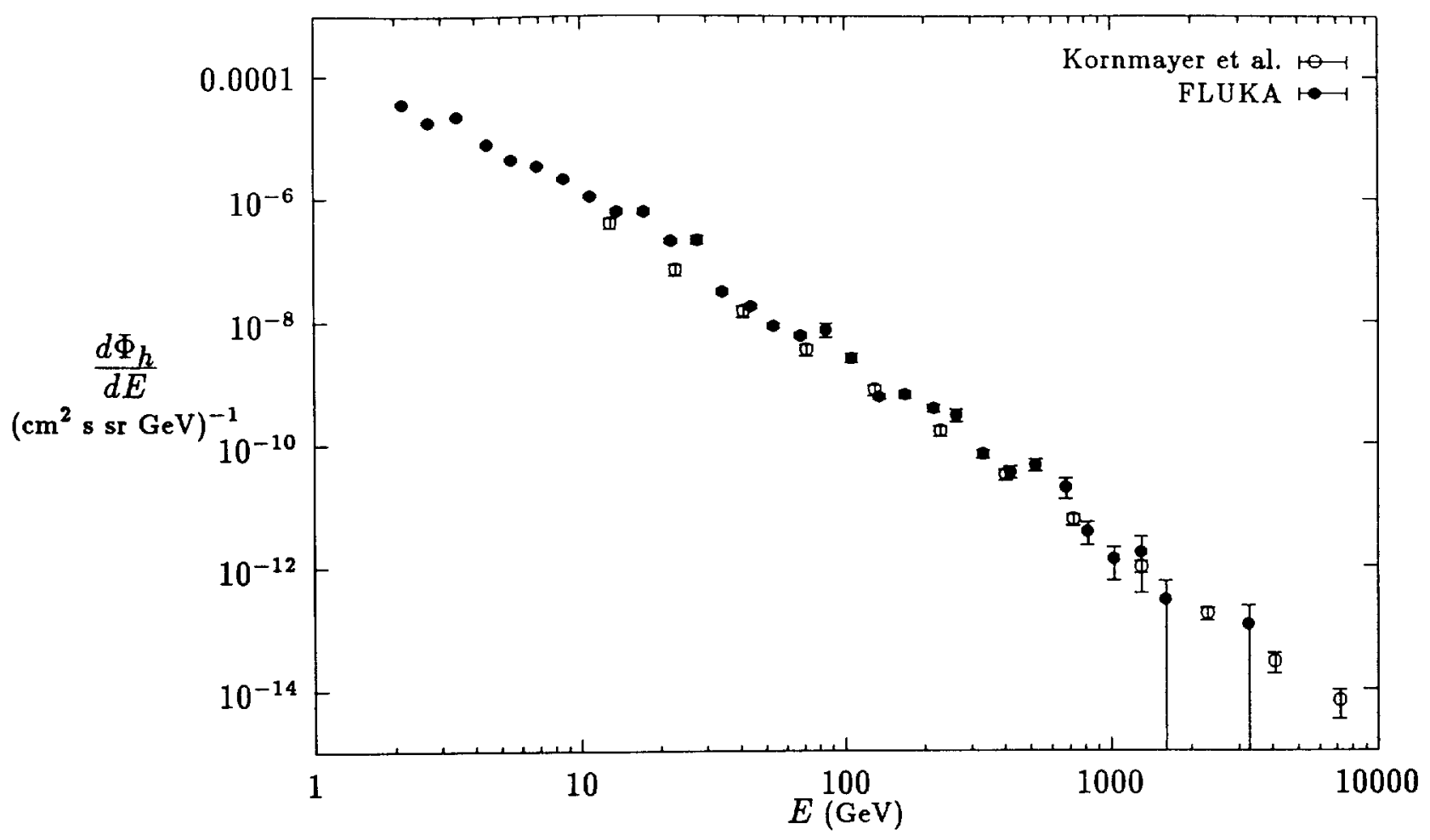

a)

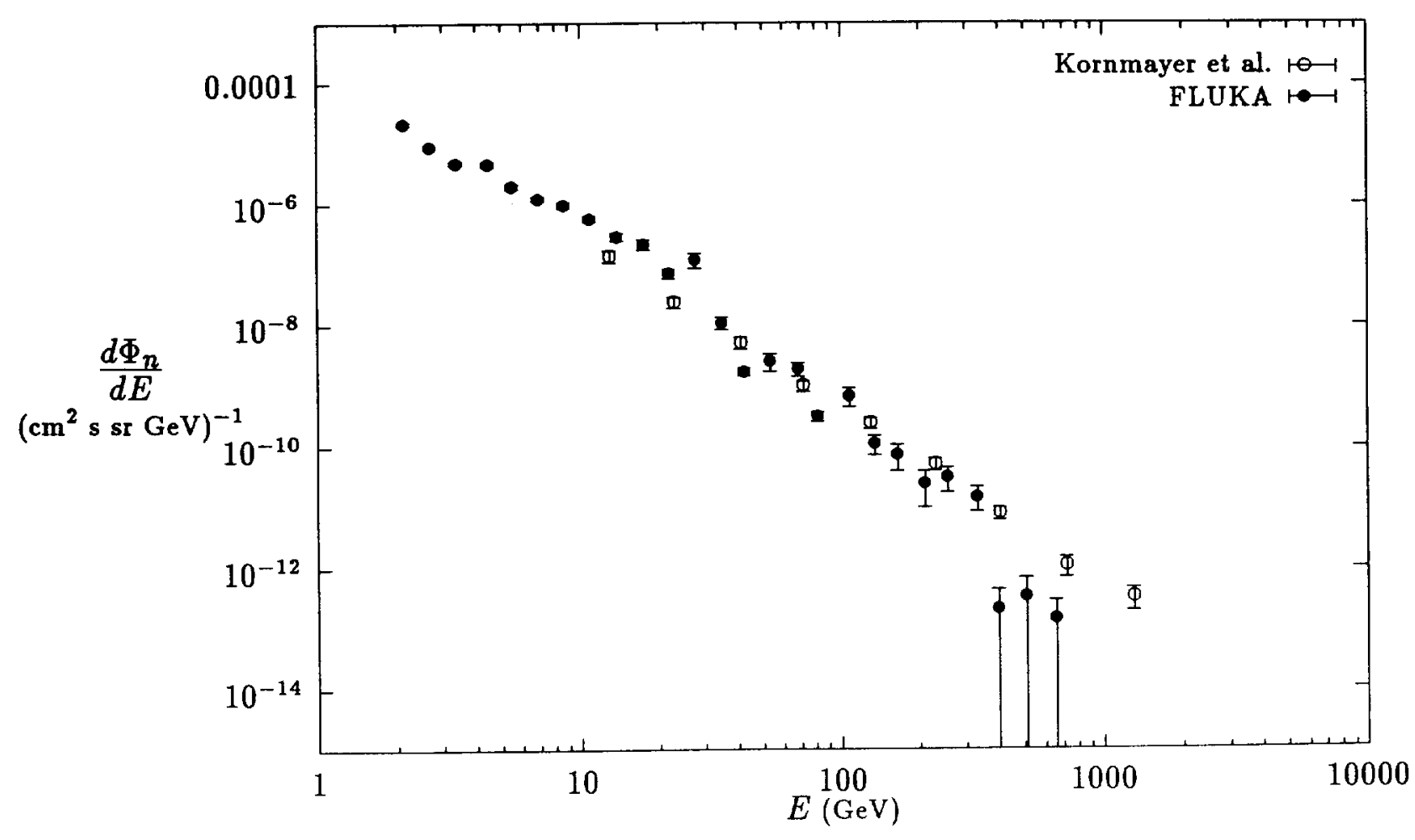

b)

Fig. 11 


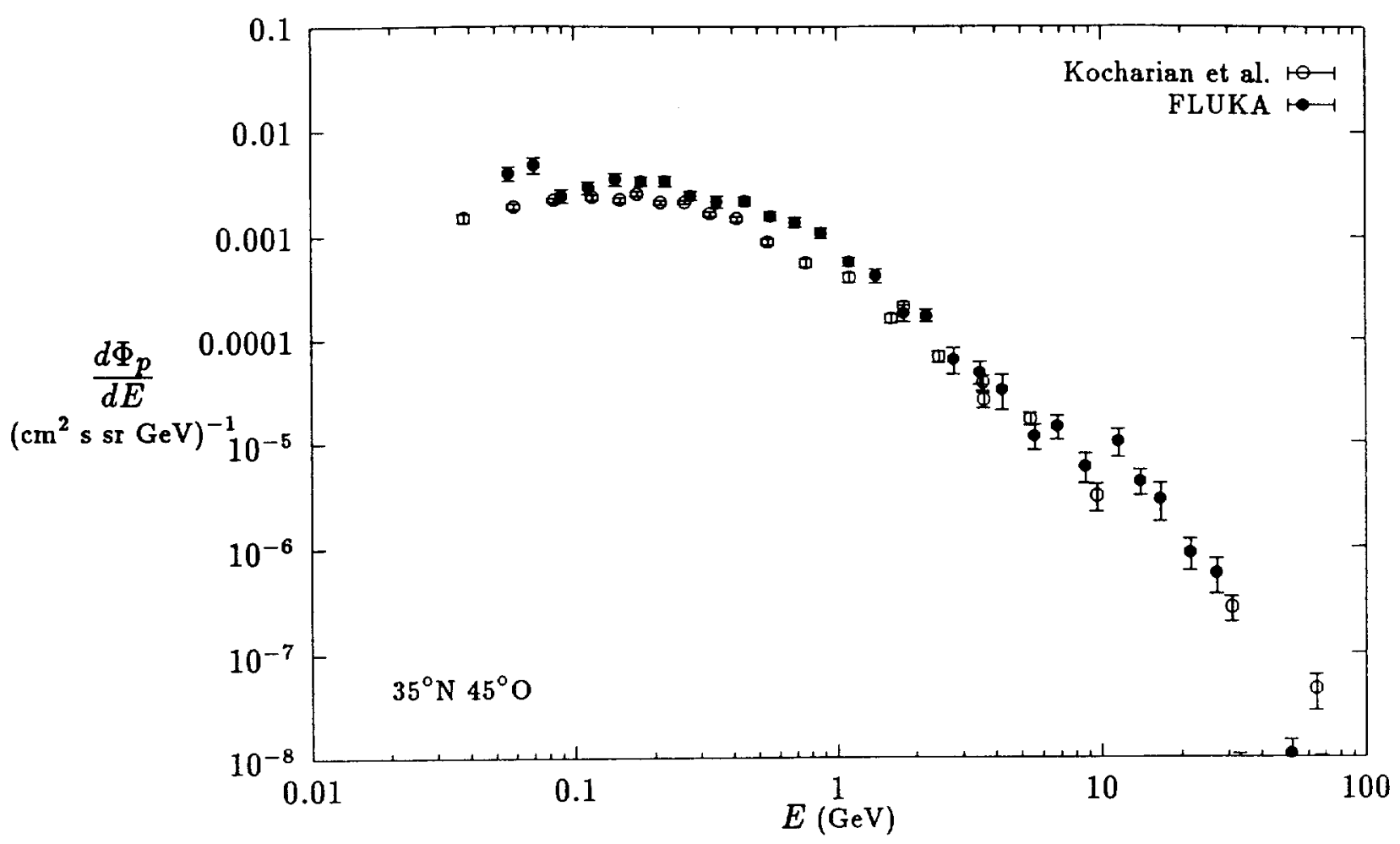

a)

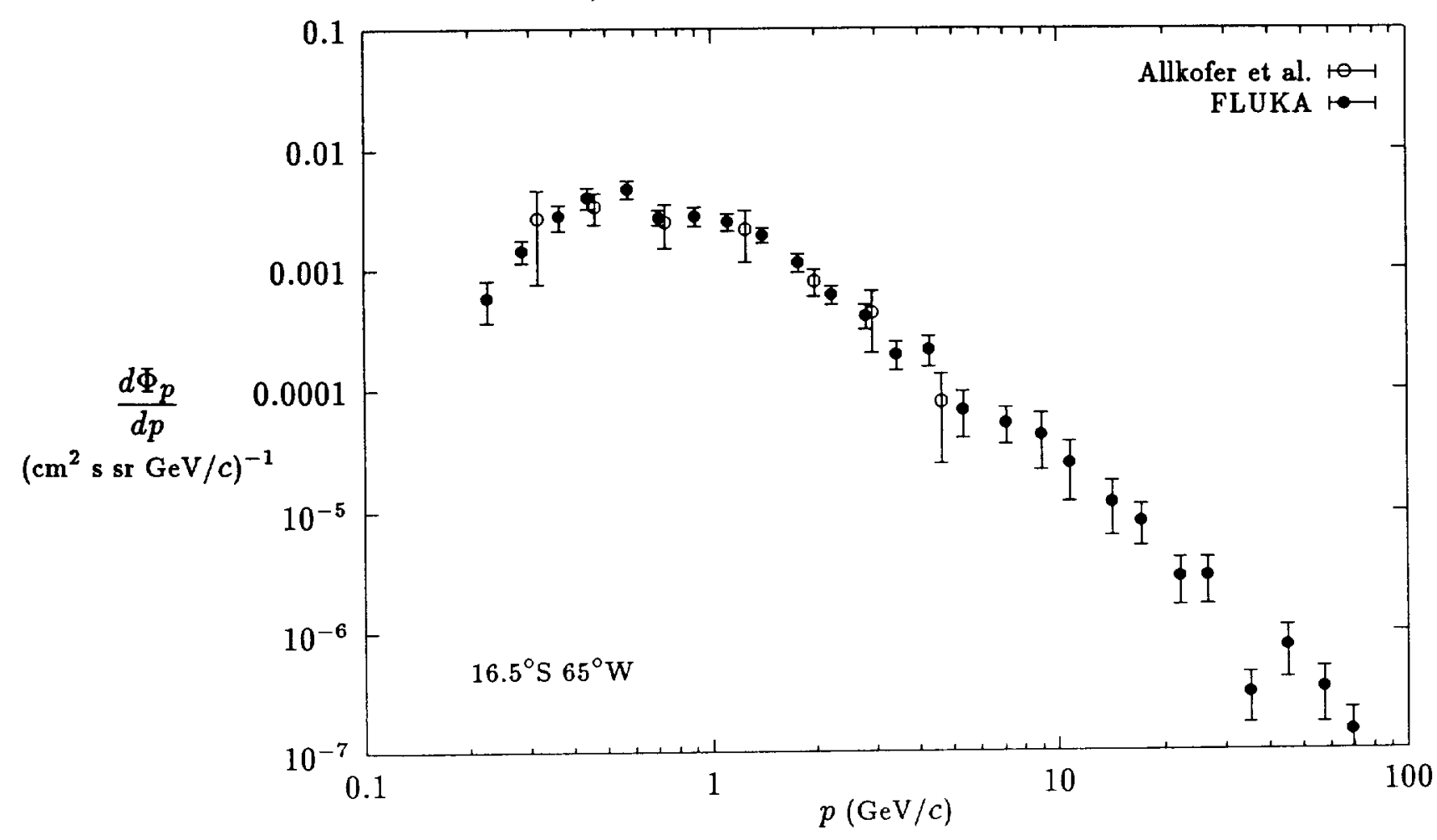

b)

Fig. 12 